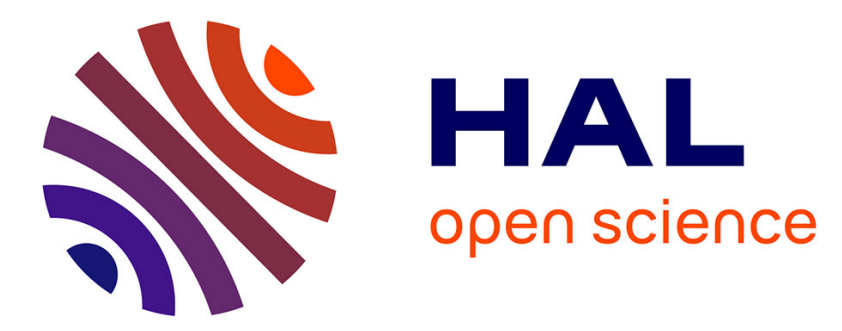

\title{
Multistability and oscillations in genetic control of metabolism
}

Diego A. Oyarzún, Madalena Chaves, Marit Hoff-Hoffmeyer-Zlotnik

\section{To cite this version:}

Diego A. Oyarzún, Madalena Chaves, Marit Hoff-Hoffmeyer-Zlotnik. Multistability and oscillations in genetic control of metabolism. Journal of Theoretical Biology, 2012, 295, pp.139-153. 10.1016/j.jtbi.2011.11.017 . hal-00848440

\section{HAL Id: hal-00848440 \\ https://hal.inria.fr/hal-00848440}

Submitted on 9 Aug 2013

HAL is a multi-disciplinary open access archive for the deposit and dissemination of scientific research documents, whether they are published or not. The documents may come from teaching and research institutions in France or abroad, or from public or private research centers.
L'archive ouverte pluridisciplinaire HAL, est destinée au dépôt et à la diffusion de documents scientifiques de niveau recherche, publiés ou non, émanant des établissements d'enseignement et de recherche français ou étrangers, des laboratoires publics ou privés. 
This is a preliminary version of the article published as:

D.A. Oyarzún, M. Chaves and M. Hoff-Hoffmeyer-Zlotnik, J. Theor. Biol., 295, pp. 139-153, 2012.

\title{
Multistability and oscillations in genetic control of metabolism
}

\author{
Diego A. Oyarzún* Madalena Chaves ${ }^{\dagger} \quad$ Marit Hoff-Hoffmeyer-Zlotnik ${ }^{\ddagger}$
}

\begin{abstract}
Genetic control of enzyme activity drives metabolic adaptations to environmental changes, and therefore the feedback interaction between gene expression and metabolism is essential to cell fitness. In this paper we develop a new formalism to detect the equilibrium regimes of an unbranched metabolic network under transcriptional feedback from one metabolite. Our results indicate that one-to-all transcriptional feedback can induce a wide range of metabolic phenotypes, including mono-, multistability and oscillatory behavior. The analysis is based on the use of switch-like models for transcriptional control and the exploitation of the time scale separation between metabolic and genetic dynamics. For any combination of activation and repression feedback loops, we derive conditions for the emergence of a specific phenotype in terms of genetic parameters such as enzyme expression rates and regulatory thresholds. We find that metabolic oscillations can emerge under uniform thresholds and, in the case of operoncontrolled networks, the analysis reveals how nutrient-induced bistability and oscillations can emerge as a consequence of the transcriptional feedback.
\end{abstract}

\section{Introduction}

Metabolism and gene expression are two fundamental levels of cellular regulation. They are tightly interconnected, as gene expression can impact metabolic activity through changes in enzyme concentrations and, conversely, metabolic species can control gene transcription and modulate enzyme expression. These two levels have specific functions and properties, and it remains a challenge to identify the properties that emerge from their interaction $[1,2]$. Metabolic-genetic interactions can lead to a diverse range of dynamic behaviors, each one of which defines a specific metabolic phenotype. Our understanding of natural regulatory circuits is important not only for revealing the design principles that underlie observed metabolic dynamics, but also for our ability to design synthetic circuits that enable new phenotypes [3].

A number of recent studies have demonstrated the importance of crosstalk interactions between genetic and metabolic systems. For example, the works in $[4,5]$ developed integrated metabolic-genetic models for catabolite repression in E. coli and the central metabolism of B. subtilis, respectively, whereas transcriptional regulatory principles were discussed in $[1,6]$. These studies have focused on large scale models that allow for useful simulation-based predictions, but their complexity hinders the analysis of the mechanisms by which metabolic phenotypes emerge from the interconnection between the metabolic and genetic domains.

A specific metabolic phenotype depends on the regulatory topology, which defines which metabolites regulate which enzymes, and the regulatory logic, which specifies whether a metabolite activates or represses gene expression. The main objective of this paper is to investigate the phenotypes generated by a one-to-all regulatory topology under different configurations of activation and repression feedback loops.

\footnotetext{
${ }^{*}$ Corresponding author. Centre for Synthetic Biology and Innovation, Department of Bioengineering, Imperial College London, SW7 2AZ, United Kingdom. Email: d.oyarzun@imperial.ac.uk.

${ }^{\dagger}$ BIOCORE, INRIA, 2004 Route des Lucioles, BP 93, 06902 Sophia Antipolis, France. Email: madalena.chaves@inria.fr.

${ }^{\ddagger}$ Department of Nature and Engineering, University of Applied Sciences Bremen, Neustadtswall 30, 28199 Bremen, Germany. Email: mzlotnik@stud.hs-bremen.de.
} 
In a one-to-all topology, a single metabolic species modulates the activity of all enzymes via metaboliteresponsive transcription factors. One-to-all regulatory motifs are also referred to as "single input modules" and were identified as one of the building blocks in genome-wide bacterial networks [7]. Metabolic networks under one-to-all transcriptional regulation appear in uptake and utilization/biosynthesis systems, whereby enzyme expression is controlled by intracellular metabolites, as in the case of the lactose operon [8, 9], and amino acid biosynthesis, e.g. the tryptophan operon [10] and the arginine synthesis network [11].

We focus on a model that integrates classical kinetic equations for metabolite dynamics and piecewise affine (PA) differential equations to describe switch-like transcriptional regulation exerted by the metabolite (Section 2). The metabolic subsystem describes the evolution of $n$ metabolites through a chain of $(n+$ 1) enzymatic reactions with a generic class of enzyme kinetics that includes Michaelis-Menten and Hill equation as special cases. The genetic circuit models enzyme concentrations in response to the back-fed metabolite and can account for any combination of activation or repression regulatory loops.

The use of PA models for biochemical systems was pioneered by Glass and Kaufmman [12] and has lead to a number of extensions $[13,14,15,16]$ and the development of dedicated simulation tools [17]. They provide a convenient way of encoding switch-like regulation with a small parameter set (i.e. only expression rates and regulatory thresholds). The analysis of PA models, however, has been limited to purely genetic networks and their impact on protein concentrations. In this paper we develop a new framework to analyze a PA genetic system coupled with a metabolic network. Our specific goal is to identify what types of metabolic phenotypes can appear and how they depend on the gene regulatory circuit. The main contributions of this work are:

Model reduction and analysis We show that under a time scale separation the complete system reduces to a planar PA system defined in three conic domains (Section 3). The conic geometry of the reduced system contrasts with PA systems for purely genetic systems (which are defined on rectangular grids $[12,14,15])$, and is a consequence of the metabolic-genetic crosstalk. The 3-cone model can be studied as a pair of 2-cone ones, and therefore we provide a rigorous analysis of a PA system in a 2-cone partition. To this end we use Filippov's construction for discontinuous dynamical systems [18] and establish geometric conditions for the existence multiple equilibria and limit cycles (Section 4).

Multistability and oscillatory behavior We use the derived conditions to detect multiple equilibria and oscillations in the metabolic network subject to one-to-all transcriptional feedback (Section 5). The analysis suggests that one-to-all regulation can generate a wide range of metabolic phenotypes: mono-, multistability, and oscillatory behavior. For given metabolic network and regulatory logic, a particular phenotype appears as a function of gene expression parameters and enzyme degradation rates. We observe that under different activation or repression thresholds, the regulatory circuit can exhibit multiple equilibrium fluxes, whereas oscillatory behavior can emerge under identical thresholds.

Operon regulation We apply our methodology to the special case of bacterial operons, whereby a set of genes are collectively controlled by a single transcription factor (Section 6). The analysis predicts nutrientinduced bistability, which was experimentally observed in the lactose operon [19], and also suggests the emergence of nutrient-induced oscillations.

\section{Generic model for an unbranched metabolic network under tran- scriptional regulation}

We consider an unbranched metabolic network under one-to-all transcriptional regulation from a metabolite. A schematic diagram of such class of networks is shown in Figure 1A, where $s_{i}$ denotes the concentra- 
tion of the $i^{\text {th }}$ metabolite and $v_{i}$ is the rate of the $i^{\text {th }}$ reaction (catalyzed by an enzyme with concentration $\left.e_{i}\right)$.

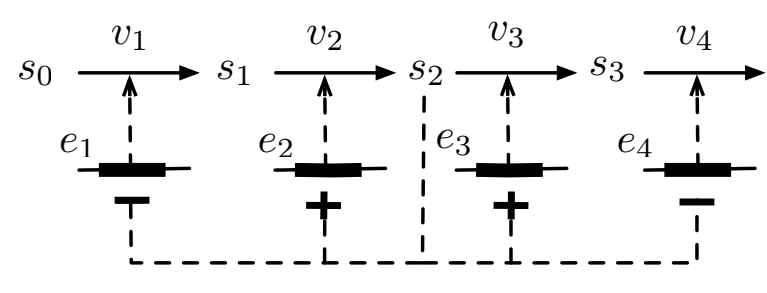

A

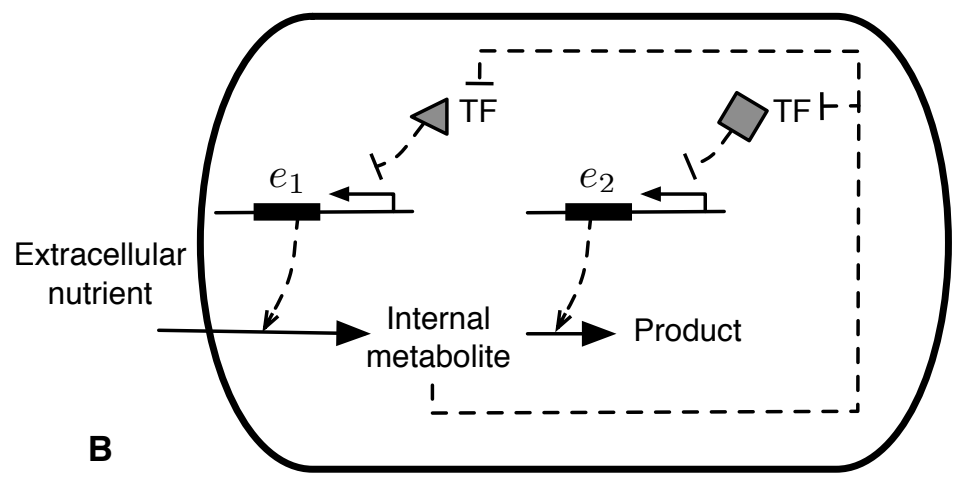

Figure 1: One-to-all gene regulation in an unbranched metabolic network. (A) Generic model. (B) Example network where nutrient uptake and consumption are controlled by metabolite-responsive transcription factors $(\mathrm{TF})$.

As a way of accounting for the mass exchange between the network and its environment, we assume that the metabolic substrate $s_{0}$ is constant. For the sake of generality, in this paper we deal with networks of $n$ metabolites and $n+1$ enzymes regulated by metabolite $s_{\ell-1}(\ell>1)$. The rate of change of both metabolite and enzyme concentrations can be described by the differential equations

$$
\begin{aligned}
& \dot{s}_{i}=v_{i}\left(s_{i-1}, e_{i}\right)-v_{i+1}\left(s_{i}, e_{i+1}\right), \\
& \dot{e}_{i}=\kappa_{i}^{0}+\kappa_{i}^{1} \sigma_{i}\left(s_{\ell-1}, \theta_{i}\right)-\gamma_{i} e_{i} .
\end{aligned}
$$

where $\kappa_{i}^{0}, \kappa_{i}^{1}, \theta_{i}, \gamma_{i}$ are positive parameters. The metabolic model (1) arises from the mass balance between the reactions that produce and consume $s_{i}$, whereas the model for the enzyme concentrations (2) comes from the balance between protein synthesis and degradation (modeled as a linear process with kinetic constant $\gamma_{i}$ ). The constant $\kappa_{i}^{0}$ represents a basal expression level of protein $e_{i}$, whereas $\kappa_{i}^{1}$ and the functions $\sigma_{i}$ model the effect of the regulator on the synthesis rates.

The regulatory function $\sigma_{i}\left(s_{\ell-1}, \theta_{i}\right)$ represents the lumped effect of gene expression control by a transcription factor, together with its interaction with the regulator $s_{\ell-1}$. This kind of transcriptional regulation appears, for example, in bacterial uptake and utilization systems, whereby enzyme expression is controlled by metabolite-responsive transcription factors (Figure 1B), e.g. the lactose operon [8, 9], and also in aminoacid biosynthesis, e.g. the tryptophan operon [10] and arginine synthesis [11].

To account for the typical switch-like nature of transcriptional regulation, we take $\sigma_{i}$ to be a step function; depending on whether gene expression is activated or repressed by $s_{\ell-1}$, we assign $\sigma_{i}=\sigma^{+}$or $\sigma_{i}=\sigma^{-}=1-\sigma^{+}$, respectively, with

$$
\sigma^{+}\left(s_{\ell-1}, \theta\right)=\left\{\begin{array}{ll}
0, & s_{\ell-1}<\theta \\
1, & s_{\ell-1}>\theta
\end{array} .\right.
$$

This class of regulatory functions is widely used in the analysis of genetic networks $[20,15]$ and was first suggested in [12]. Under this regulatory model, genes can be switched ON or OFF depending on the metabolic regulator: in the OFF state gene $i$ is transcribed at a constitutive rate $\kappa_{i}^{0}$ and the protein concentration will approach

$$
E_{i}^{\mathrm{off}}=\kappa_{i}^{0} / \gamma_{i}
$$


whereas in the ON state its transcription rate jumps to $\kappa_{i}^{0}+\kappa_{i}^{1}$ and the concentration approaches

$$
E_{i}^{\mathrm{on}}=\left(\kappa_{i}^{0}+\kappa_{i}^{1}\right) / \gamma_{i}
$$

The enzyme kinetics are comprised in the reaction rates $v_{i}\left(s_{i-1}, e_{i}\right)$, and in the sequel we will not presuppose a specific form for them. Instead, to keep the analysis as general as possible, we make the following generic assumption on the enzyme kinetics.

Assumption 1 The metabolic reaction rates are linear in the enzyme concentrations and non-decreasing functions of the metabolite concentrations. The enzyme kinetics can then be written as

$$
v_{i}\left(s_{i-1}, e_{i}\right)=g_{i}\left(s_{i-1}\right) e_{i}
$$

where $g_{i}$ is the enzyme turnover rate (i.e. the reaction rate per unit of enzyme concentration) and satisfies

$$
\frac{\partial g_{i}\left(s_{i-1}\right)}{\partial s_{i-1}} \geq 0
$$

The monotonicity condition in (7) accounts for a broad class of saturable enzyme kinetics that includes, in particular, Michaelis-Menten and Hill kinetics [21]. The saturable form of the enzyme kinetics limits the parameter space that yields a valid equilibrium. This is discussed in Appendix A. Our aim in the rest of the paper is to characterize the dynamic properties of the regulatory circuit, including the detection of multiple equilibria and oscillatory behavior.

\section{Model reduction}

\subsection{Quasi steady state approximation}

Metabolic dynamics operate in a much shorter time scale than their genetic counterpart [22]. This property allows for the approximation of the nonlinear dynamics in (1) by an algebraic relationship between the enzymes and metabolite concentrations. If the metabolites are assumed to be in quasi steady state (QSS) with respect to the enzyme concentrations, then we set $\dot{s}_{i}(t)=0$ for all $t \geq 0$ to obtain

$$
g_{i+1}\left(s_{i}(t)\right)=g_{i}\left(s_{i-1}(t)\right) \frac{e_{i}(t)}{e_{i+1}(t)},
$$

Equation (8) holds for every $i=1,2, \ldots, n$ and hence it is equivalent to

$$
g_{i}\left(s_{i-1}(t)\right)=g_{1}\left(s_{0}\right) \frac{e_{1}(t)}{e_{i}(t)},
$$

for $i=2,3, \ldots, n$. The above is an algebraic equation for the metabolites as a function of the enzyme levels. The existence of a solution to (9) is discussed in Appendix A and depends on the initial conditions and the saturation values of the enzyme kinetics. From the approximation in (9), we can compute the trajectory of the regulator by solving the equation

$$
g_{\ell}\left(s_{\ell-1}(t)\right)=g_{1}\left(s_{0}\right) \frac{e_{1}(t)}{e_{\ell}(t)} .
$$

A key aspect of this approximation is that the solution of (10) depends only on two enzymes. The dynamics of the complete feedback system can thus be characterized with the 2-dimensional phase plane of the differential equations

$$
\begin{aligned}
& \dot{e}_{1}=\kappa_{1}^{0}+\kappa_{1}^{1} \sigma_{1}\left(s_{\ell-1}, \theta_{1}\right)-\gamma_{1} e_{1}, \\
& \dot{e}_{\ell}=\kappa_{\ell}^{0}+\kappa_{\ell}^{1} \sigma_{\ell}\left(s_{\ell-1}, \theta_{\ell}\right)-\gamma_{\ell} e_{\ell},
\end{aligned}
$$

subject to $s_{\ell-1}$ satisfying $(10)$. Note that in the case of product feedback $\left(s_{\ell-1}=s_{n}\right)$, the above approximation also applies to reversible metabolic reactions. 


\subsection{Reduction to a piecewise affine system in conic domains}

The algebraic equation in (10) can be interpreted as a mapping from $\mathbb{R}_{\geq 0}$ to $\mathbb{R}_{\geq 0}^{2}$, whereby each value of the regulator $s_{\ell-1}$ maps into a half-line in the $\left(e_{1}, e_{\ell}\right)$ plane. Moreover, as a consequence of the monotonicity of $g_{\ell}$, the partition of $\mathbb{R}_{\geq 0}$ induced by the thresholds can be mapped into a partition of $\mathbb{R}_{\geq 0}^{2}$ : if $s_{\ell-1}<\theta_{i}$ then

$$
g_{\ell}\left(s_{\ell-1}\right)<g_{\ell}\left(\theta_{i}\right)
$$

which combined with (10) yields

$$
e_{\ell}>\beta_{i} e_{1}
$$

with $\beta_{i}=g_{1}\left(s_{0}\right) / g_{\ell}\left(\theta_{i}\right)$. The relation in (13) defines a cone in the $x=\left(e_{1}, e_{\ell}\right)$ plane

$$
D_{i}=\left\{x \in \mathbb{R}_{\geq 0}^{2}: x_{2}>\beta_{i} x_{1}\right\}
$$

and we define its complementary cone as $\bar{D}_{i}=\mathbb{R}_{\geq 0}^{2} \backslash\left(D_{i} \cup S_{i}\right)$ with $S_{i}$ the half-line

$$
S_{i}=\left\{x \in \mathbb{R}_{\geq 0}^{2}: x_{2}=\beta_{i} x_{1}\right\}
$$

The half-line $S_{i}$ is a subset of the $\left(e_{1}, e_{\ell}\right)$ plane where the regulator reaches the switching threshold $\theta_{i}$. The dynamics of the reduced system in (11) depend on the value of $s_{\ell-1}$ with respect to the thresholds $\theta_{1}$ and $\theta_{\ell}$. Assume, without loss of generality, that $\theta_{1}<\theta_{\ell}$ (the problem can be treated analogously in the case $\theta_{1}>\theta_{\ell}$, and the case $\theta_{1}=\theta_{\ell}$ can be treated as later in Section 4$)$. With the previous definitions we can establish the following relations

$$
\begin{aligned}
s_{\ell-1}<\theta_{1} & \Longleftrightarrow x \in R_{1}, \\
\theta_{1}<s_{\ell-1}<\theta_{\ell} & \Longleftrightarrow x \in R_{1 \ell}, \\
s_{\ell-1}>\theta_{\ell} & \Longleftrightarrow x \in R_{\ell},
\end{aligned}
$$

where $R_{1}=D_{1}, R_{1 \ell}=\bar{D}_{1} \cap D_{\ell}$ and $R_{\ell}=\bar{D}_{\ell}$. In the sequel we refer to $S_{i}$ as a switching domain, whereas the cones $R_{j}$ are called regular domains (see [15] for detailed definitions). The system in (11) is equivalent to a piecewise affine (PA) system [23] in three conic domains

$$
\dot{x}=h(x)-\Gamma x,
$$

where

$$
h(x)=\left\{\begin{array}{ll}
h^{1} & x \in R_{1} \\
h^{1 \ell} & x \in R_{1 \ell}, \\
h^{\ell} & x \in R_{\ell}
\end{array} \quad \Gamma=\left[\begin{array}{cc}
\gamma_{1} & 0 \\
0 & \gamma_{\ell}
\end{array}\right] .\right.
$$

The vectors $h^{1}, h^{1 \ell}$ and $h^{\ell}$ are constant and their values depend on whether $s_{\ell-1}$ activates or represses the expression of enzymes $e_{1}$ and $e_{\ell}$. For example, in the case of repression of both enzymes (i.e. $\sigma_{1}=\sigma^{-}$and $\sigma_{\ell}=\sigma^{-}$) we have

$$
h^{1}=\left[\begin{array}{l}
\kappa_{1}^{0}+\kappa_{1}^{1} \\
\kappa_{\ell}^{0}+\kappa_{\ell}^{1}
\end{array}\right], \quad h^{1 \ell}=\left[\begin{array}{c}
\kappa_{1}^{0} \\
\kappa_{\ell}^{0}+\kappa_{\ell}^{1}
\end{array}\right], \quad h^{\ell}=\left[\begin{array}{l}
\kappa_{1}^{0} \\
\kappa_{\ell}^{0}
\end{array}\right]
$$


wheres in the case of activation (i.e. $\sigma_{1}=\sigma^{+}$and $\sigma_{\ell}=\sigma^{+}$):

$$
h^{1}=\left[\begin{array}{l}
\kappa_{1}^{0} \\
\kappa_{\ell}^{0}
\end{array}\right], \quad h^{1 \ell}=\left[\begin{array}{c}
\kappa_{1}^{0}+\kappa_{1}^{1} \\
\kappa_{\ell}^{0}
\end{array}\right], \quad h^{\ell}=\left[\begin{array}{c}
\kappa_{1}^{0}+\kappa_{1}^{1} \\
\kappa_{\ell}^{0}+\kappa_{\ell}^{1}
\end{array}\right]
$$

These vectors determine the location of the focal points of the PA system, defined as

$$
\phi^{1}=\Gamma^{-1} h^{1}, \quad \phi^{1 \ell}=\Gamma^{-1} h^{1 \ell}, \quad \phi^{\ell}=\Gamma^{-1} h^{\ell} .
$$

By definition the focal points are combinations of the ON and OFF enzyme concentrations in (4)-(5), and their particular location depends exclusively on the type of feedback regulation. Figure 2 shows the possible locations of the focal points for each combination of transcriptional regulation. In what follows we will assume that the focal points do not lie in the switching domains. For any $x\left(t_{0}\right)$ in a regular domain, e.g. $x\left(t_{0}\right) \in R_{1}$, the right-hand side of (17) is well defined and its solution satisfies a standard affine differential equation, that is

$$
x(t)=\phi^{1}+\mathrm{e}^{\Gamma\left(t_{0}-t\right)}\left(x\left(t_{0}\right)-\phi^{1}\right), t \geq t_{0}
$$

so that $x(t)$ monotonically approaches $\phi^{1}$, possibly reaching the switching domain $S_{1}$, where the vector field of (17) is not defined, and thus a specialized analysis is required. As we shall see in the next section, the location of the focal points plays a major role in the dynamics of (17).

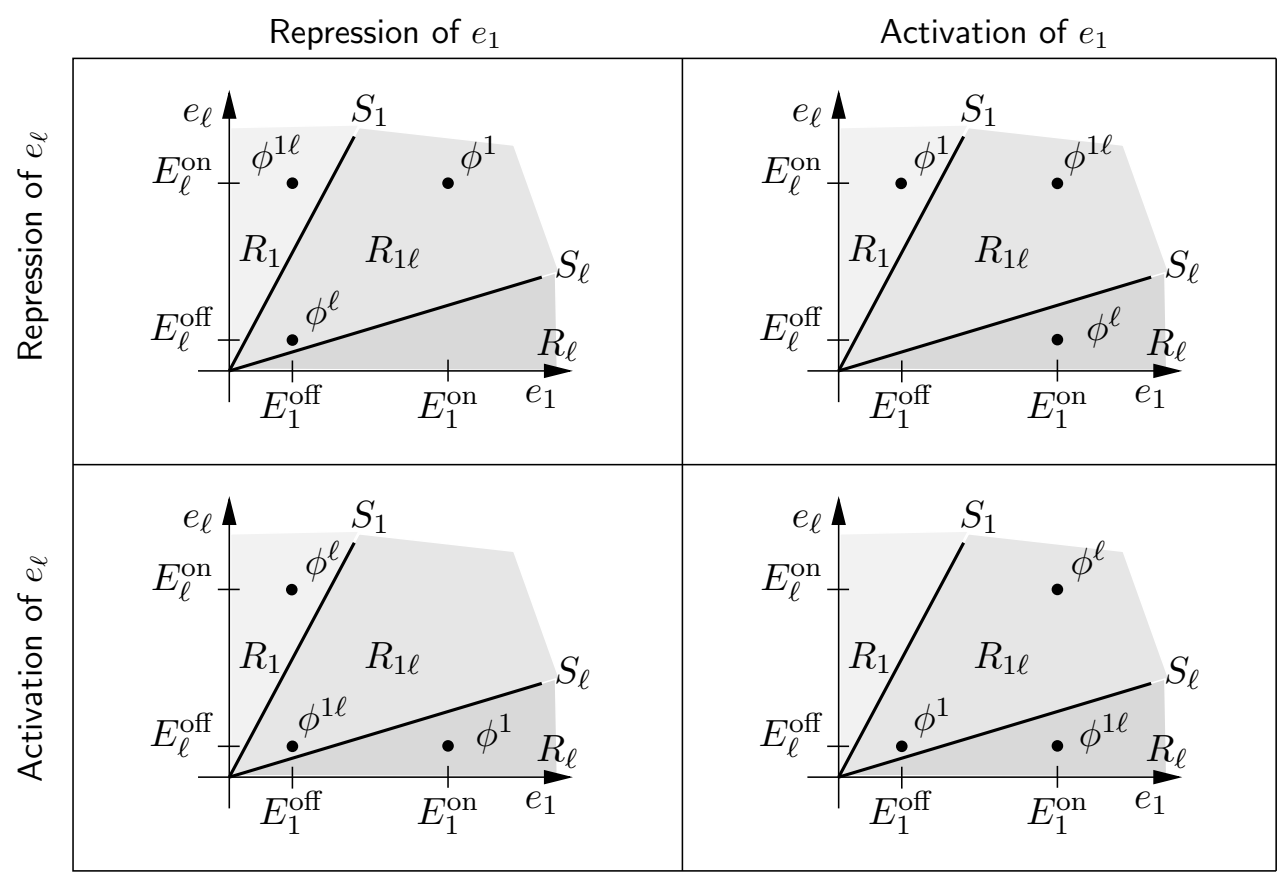

Figure 2: Conic partition of the enzyme state space (for the case $\theta_{1}<\theta_{\ell}$ ) and location of the focal points for different combinations of activation and repression loops.

\section{Identical regulatory thresholds: the two cone case}

We first focus on the case when the regulatory thresholds of enzymes $e_{1}$ and $e_{\ell}$ are identical. This case is simpler because if $\theta_{1}=\theta_{\ell}$, the cone $R_{1 \ell}$ in Figure 4 vanishes and the system is defined only in two cones. 
In this section we state the fundamental properties of a 2-cone PA system, which we will use later (Section 5) for the more general 3-cone case that appears under different regulatory thresholds. Our analysis of the 2-cone problem has been partly reported in [24], and we have included the proofs in Appendix B.

Here we use a more general notation and consider the PA system:

$$
\dot{x}=\left\{\begin{array}{ll}
f(x) & x \in D_{f} \\
g(x) & x \in D_{g}
\end{array},\right.
$$

with $f(x)=\left[f_{1}(x) f_{2}(x)\right]^{T}=h^{f}-\Gamma x$, and $g(x)=\left[\begin{array}{ll}g_{1}(x) & g_{2}(x)\end{array}\right]^{T}=h^{g}-\Gamma x$, respectively. The vectors $h^{f}$ and $h^{g}$ are entrywise nonnegative and in terms of the notation of (17), we have $h^{f}=h^{1}, h^{g}=h^{\ell}$ with focal points $\phi^{f}=\phi^{1}, \phi^{g}=\phi^{\ell}$, and two cones $D_{f}=R_{1}$ and $D_{g}=R_{\ell}$ separated by the half-line $S=S_{1}=S_{\ell}$ with slope $\beta=\beta_{1}=\beta_{\ell}$. Previous studies of the 2-cone problem can be found in [25], but their results are limited to the case where $h^{g}=-h^{f}$, and therefore they are not directly applicable to our case.

\subsection{Geometric definitions}

For the forthcoming analysis it is convenient to define the sets

$$
\begin{aligned}
& \Omega_{f}^{-}=\left\{x \in D_{f} \cup S: f_{2}(x)-\beta f_{1}(x) \leq 0\right\}, \\
& \Omega_{g}^{-}=\left\{x \in D_{g} \cup S: g_{2}(x)-\beta g_{1}(x) \leq 0\right\},
\end{aligned}
$$

and $\Omega_{f}^{+}=\left(D_{f} \cup S\right) \backslash \Omega_{f}^{-}, \Omega_{g}^{+}=\left(D_{g} \cup S\right) \backslash \Omega_{g}^{-}$, respectively. Alternatively, we can construct these sets by defining the normal vector of $S$ as $\eta^{\perp}=\left[\begin{array}{ll}-\beta & 1\end{array}\right]^{T}$, so that, for example, the set $\Omega_{f}^{-}$contains all points satisfying $\left\langle f(x), \eta^{\perp}\right\rangle \leq 0$, or equivalently $\left\langle\Gamma\left(x-\phi^{f}\right), \eta^{\perp}\right\rangle \geq 0$. Analogous definitions can be constructed for the other sets in (24). The boundary between $\Omega_{f}^{-}$and $\Omega_{f}^{+}$is the half-line

$$
C_{f}=\left\{x \in \mathbb{R}_{\geq 0}^{2}:\left\langle\Gamma\left(x-\phi^{f}\right), \eta^{\perp}\right\rangle=0\right\}
$$

whereas the boundary between $\Omega_{g}^{-}$and $\Omega_{g}^{+}$is the half-line

$$
C_{g}=\left\{x \in \mathbb{R}_{\geq 0}^{2}:\left\langle\Gamma\left(x-\phi^{g}\right), \eta^{\perp}\right\rangle=0\right\} .
$$

The half-lines $C_{f}$ and $C_{g}$ are parallel with slope $\beta \gamma_{1} / \gamma_{\ell}$, and each one contains one of the focal points, i.e. $\phi^{f} \in C_{f}$ and $\phi^{g} \in C_{g}$. The intersection points $x^{f}=C_{f} \cap S$ and $x^{g}=C_{g} \cap S$ have horizontal coordinates

$$
x_{1}^{f}=\frac{\left\langle h^{f}, \eta^{\perp}\right\rangle}{\beta\left(\gamma_{\ell}-\gamma_{1}\right)}, \quad x_{1}^{g}=\frac{\left\langle h^{g}, \eta^{\perp}\right\rangle}{\beta\left(\gamma_{\ell}-\gamma_{1}\right)} .
$$

\subsection{Solutions in the switching domain}

The vector field of PA system (23) is discontinuous at the switching domain $S$; intuitively, the behavior of solutions at $S$ will depend on the relative direction of the vector fields $f$ and $g$ in a vicinity of $S$. Trajectories can: cross between cones if the vector fields point in a similar direction, slide along $S$ if the vector fields point in opposite directions towards $S$, and be repelled from $S$ is the vector fields point in opposite directions away from $S$. The last two cases are known as stable and unstable sliding motion in the literature [15]. As shown in the next three results, each one of the aforementioned cases can be characterized in terms of the $\Omega$ sets defined in (24).

Theorem 1 (Solutions in the switching domain) The solutions of (23): 
(a) cross from $D_{g}$ to $D_{f}$ in the segment $L_{g f}=\Omega_{f}^{+} \cap \Omega_{g}^{+} \subseteq S$,

(b) cross from $D_{f}$ to $D_{g}$ in the segment $L_{f g}=\Omega_{f}^{-} \cap \Omega_{g}^{-} \subseteq S$,

(c) exhibit stable sliding motion in the segment $L_{s}=\Omega_{f}^{-} \cap \Omega_{g}^{+} \subseteq S$,

(d) exhibit unstable sliding motion in the segment $L_{\bar{s}}=\Omega_{f}^{+} \cap \Omega_{g}^{-} \subseteq S$.

Moreover, define the angle $\vartheta_{1}=\angle\left(h^{f}-h^{g}, \eta^{\perp}\right)$, then

$$
\begin{aligned}
& L_{\bar{s}}=\emptyset \Longleftrightarrow \vartheta_{1} \in\left[-\pi,-\frac{\pi}{2}\right) \cup\left(\frac{\pi}{2}, \pi\right], \\
& L_{s}=\emptyset \Longleftrightarrow \vartheta_{1} \in\left(-\frac{\pi}{2}, \frac{\pi}{2}\right) .
\end{aligned}
$$

According to Theorem 1 the behavior of solutions in the switching domain depends essentially on the different intersections between the $\Omega$ sets; this can be seen in Figure 3 , whereby the sets $L_{s}$ and $L_{\bar{s}}$ are the intersection between $S$ and the band generated by the half-lines $C_{f}$ and $C_{g}$. A necessary condition for sliding motion (stable or unstable) is therefore that the band between $C_{f}$ and $C_{g}$ intersects $S$ in the positive quadrant. Moreover, the geometry in Figure 3 shows that at least one of the sets $L_{s}$ and $L_{\bar{s}}$ must be empty, which precludes the existence of stable and unstable sliding motion in the same switching domain. We can distinguish between these two scenarios with the condition for $\vartheta_{1}$ in (28)-(29). The particular case when $\vartheta_{1}= \pm \pi / 2$ is not covered by Theorem 1, and will be treated later in Section 4.4.

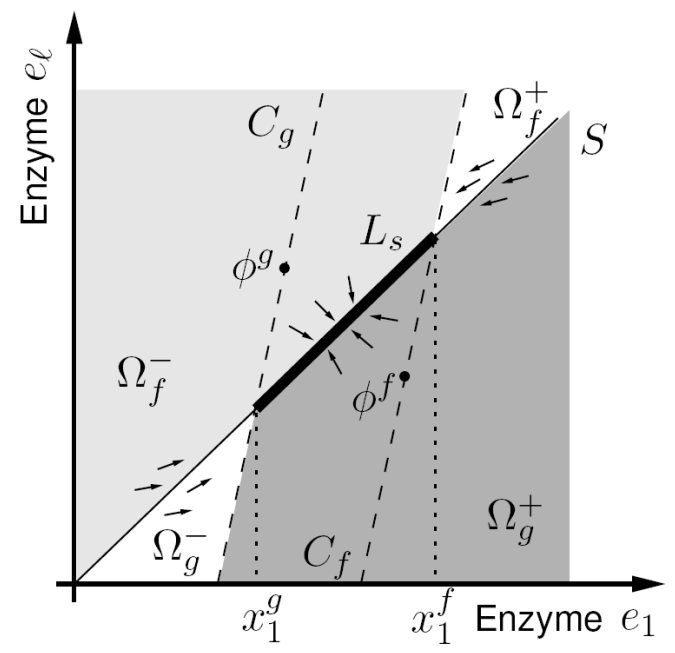

A

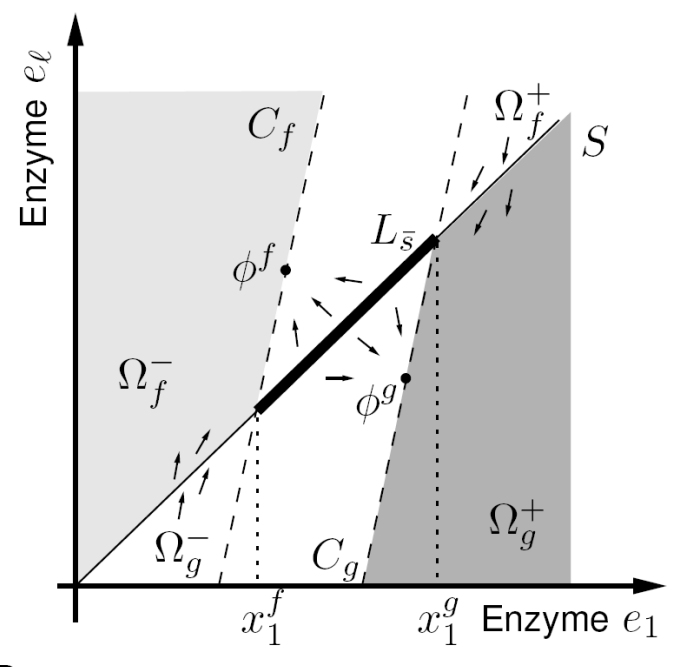

B

Figure 3: Partition of the enzyme state space for degradation rates $\gamma_{1}>\gamma_{\ell}$. Case with stable (A) and unstable (B) sliding motion.

\subsection{Equilibria}

The focal points are locally stable equilibria of the PA system provided that they belong to their respective cone, i.e. $\phi^{f} \in D_{f}$ or $\phi^{g} \in D_{g}$ (stability follows from $\gamma_{1}, \gamma_{\ell}>0$ ). In this case the focal points are referred to as regular equilibria. However, when using Filippov's method (as we did in Theorem 1, see Appendix B.1), it is possible that the trajectories reach equilibria that lie in the switching domain, which are sometimes called singular equilibria [15]. 
The existence of regular and/or singular equilibria in the PA system depend on two aspects: (a) the existence of stable or unstable sliding motion along the switching surface, and (b) whether the focal points belong to or lie outside their respective regular domain. Point (a) can be resolved with the simple angle condition in Theorem 1, but the effect of (b) requires a more detailed analysis. Since there are two focal points, two cones, and two kinds of possible sliding motion, there are eight possible configurations, all of which are studied in the next sections.

\subsubsection{Monostability with regular equilibrium.}

Four configurations are such that only one focal point is a regular equilibrium, for example, when $\phi^{f}, \phi^{g} \in$ $D_{f}$ or $\phi^{f}, \phi^{g} \in D_{g}$. In each of these four cases (and regardless of whether the sliding motion is stable or unstable), the PA system is monostable with a regular equilibrium point.

\subsubsection{Bistability with regular equilibria.}

Two configurations are such that both focal points are regular equilibria, that is, $\phi^{f} \in D_{f}$ and $\phi^{g} \in D_{g}$ with a stable sliding motion (i.e. $L_{s} \neq \emptyset$ because the angle $\vartheta_{1}$ satisfies condition (28)), or an unstable sliding motion $\left(L_{\bar{s}} \neq \emptyset\right.$ because the angle $\vartheta_{1}$ satisfies condition $\left.(29)\right)$. In these two cases, the PA system is bistable with two regular equilibria; see Figure 4A-B.

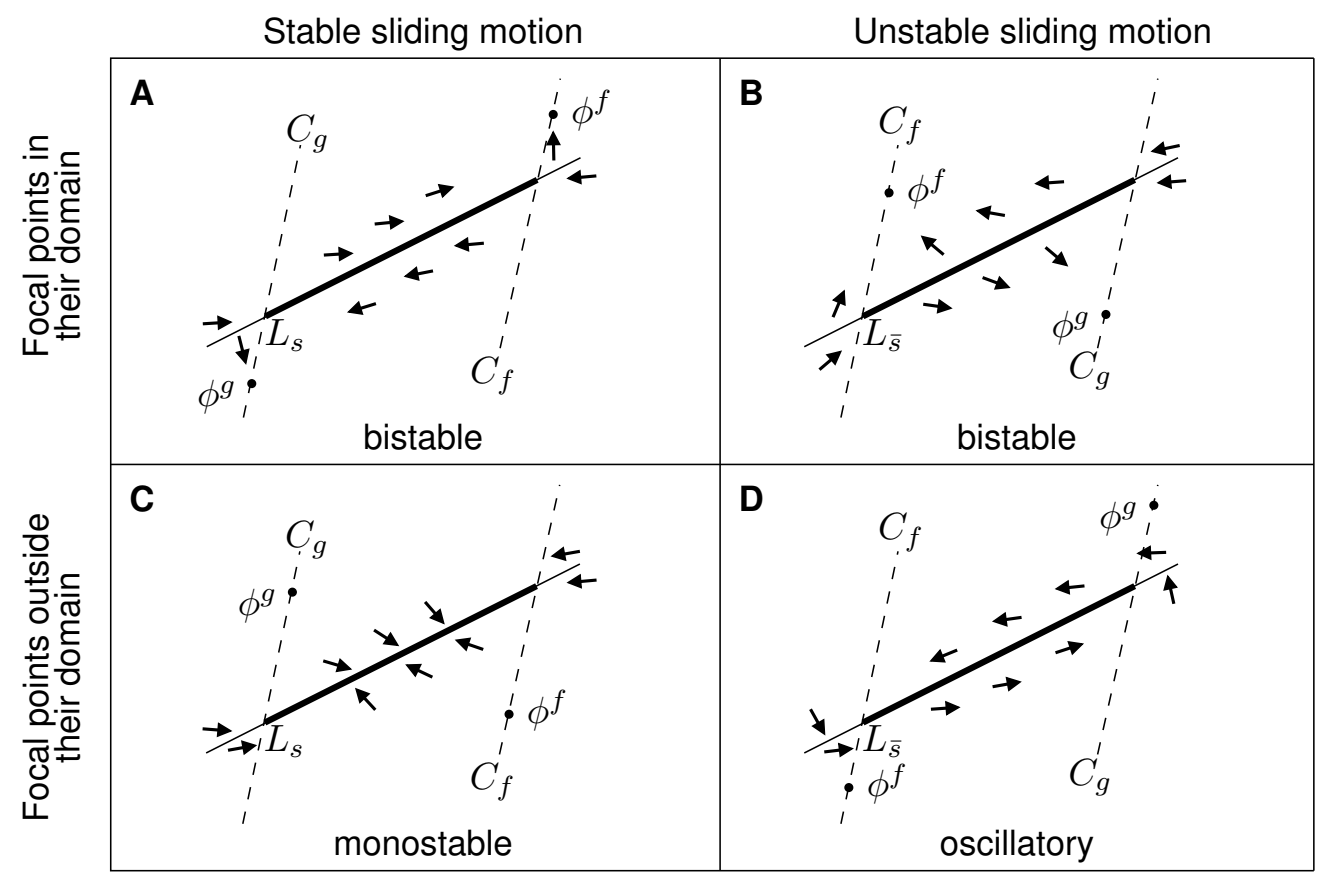

Figure 4: Mono-, bistability and oscillations in PA system defined in two conic domains. The system behavior depends on the location of the focal points and the existence of stable/unstable sliding motion. (A,B) Bistability with two regular equilibria; (C) Monostability with singular equilibrium, (D) Oscillations. Monostability with regular equilibrium not shown (see Section 4.3.1). The cases shown are for degradation rates satisfying $\gamma_{1}>\gamma_{\ell}$.

\subsubsection{Monostability with singular equilibrium.}

In the remaining two configurations both focal points lie outside their regular domains, i.e. $\phi^{f} \in D_{g}$ and $\phi^{g} \in D_{f}$. In these cases the system has a subtler behavior that depends on the type of sliding motion in $S$. 
In the case of stable sliding motion (Figure 4C), the system has a singular equilibrium, the location and stability of which are studied in the next result. On the contrary, when there exists an unstable sliding motion (Figure 4D), solutions follow stable periodic orbits, which is a topic we leave for the next section.

Theorem 2 (Singular equilibrium) Assume that $L_{s} \neq \emptyset$ and let $L_{\phi}$ be the line containing $\phi^{f}$ and $\phi^{g}$ and $\vartheta_{2}=\angle\left(\phi^{f}-\phi^{g}, \eta^{\perp}\right)$. The point

$$
\phi_{s}=L_{s} \cap L_{\phi}
$$

is a singular equilibrium of (23). Moreover, if

$$
\vartheta_{2} \in\left[-\pi,-\frac{\pi}{2}\right) \cup\left(\frac{\pi}{2}, \pi\right]
$$

then $\phi_{s}$ is locally stable, and if

$$
\vartheta_{2} \in\left(-\frac{\pi}{2}, \frac{\pi}{2}\right)
$$

then $\phi_{s}$ is unstable.

We can therefore check the existence of a singular equilibrium simply by locating the point $\phi_{s}$, whereas its local stability can be graphically checked with the condition for angle $\vartheta_{2}$. The stable case is shown in Figure 5A, and the case of an unstable singular equilibrium, shown in Figure 5B, corresponds to the bistable scenario described earlier in Section 4.3.2 and shown in Figure 4A. Moreover, in Figure 5C we observe that $L_{s} \cap L_{\phi}=\emptyset$ only when $\phi^{f}, \phi^{g} \in D_{f}$ or $\phi^{f}, \phi^{g} \in D_{g}$, and therefore Theorem 2 also accounts for the monostable case with regular equilibrium described earlier in Section 4.3.1. In the cases of Figures 5B-C, solutions may slide along $L_{s}$ but eventually escape to one of the regular domains.

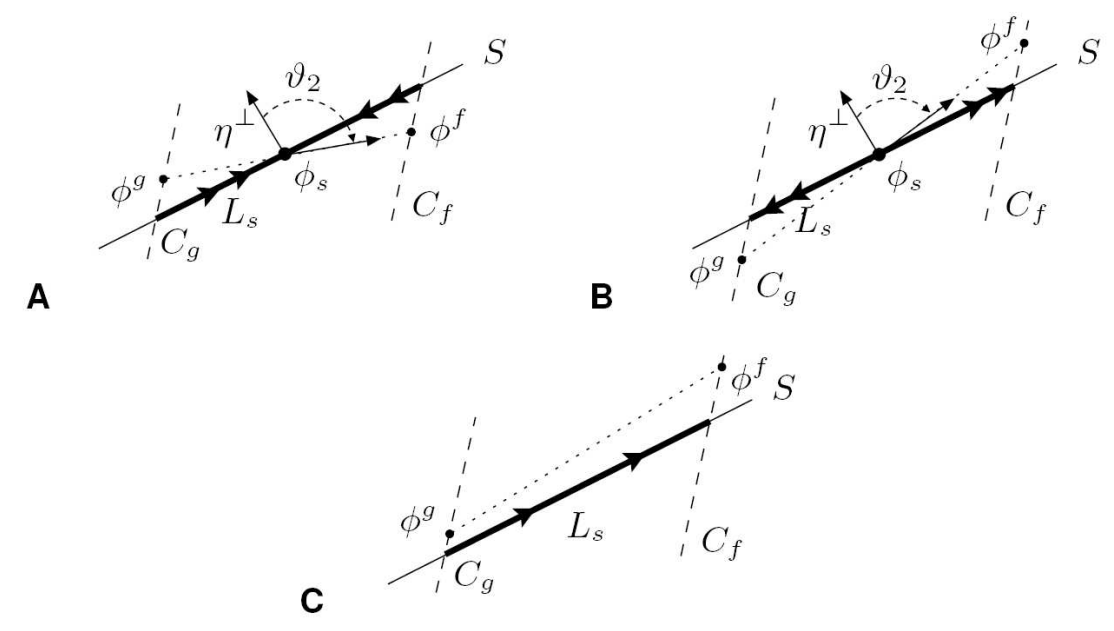

Figure 5: Existence and stability of a singular equilibrium for the PA system. (A) Stable singular equilibrium; (B) Unstable singular equilibrium; (C) No singular equilibrium. The cases shown are for degradation rates $\gamma_{1}>\gamma_{\ell}$.

\subsection{Oscillations.}

If both focal points lie outside their domain and there is unstable sliding motion (as in Figure 4D), trajectories starting in $D_{f}$ can cross to $D_{g}$ in the segment $L_{f g}$ and can cross back to $D_{f}$ in $L_{g f}$. On 
the segment $L_{\bar{s}}$ the vector fields on both $D_{g}$ and $D_{f}$ point away from $S$ and towards the interior of the domains, and therefore are pulled away from the switching domain. These qualitative observations suggest that trajectories can follow a periodic orbit around the segment $L_{\bar{s}}$, see Figure 6 . They also suggest that a region for unstable sliding motion and two crossing regions are needed for oscillations to exist; we will therefore make the following assumption.

Assumption 2 The switching domain $S$ contains: $(A)$ a region for unstable sliding motion (i.e. $L_{\bar{s}} \neq \emptyset$ ), and $(B)$ two crossing regions (i.e. $L_{g f} \neq \emptyset$ and $L_{f g} \neq \emptyset$ ).

The validity of Assumption 2A can be readily checked with the angle condition in Theorem 1, whereas Assumption 2B requires more attention. In Figure 3B we observe that the crossing domains are simultaneously nonempty only when the lines $C_{f}$ and $C_{g}$ intersect the switching domain $S$ in the positive quadrant. This is equivalent to requiring that the horizontal coordinates of $C_{f} \cap S$ and $C_{f} \cap S$ satisfy $x_{1}^{f}>0$ and $x_{1}^{g}>0$, respectively. These positivity conditions are met when (recall the expressions in (27)):

$$
\begin{aligned}
& \gamma_{1}>\gamma_{\ell},\left\langle h^{f}, \eta^{\perp}\right\rangle<0,\left\langle h^{g}, \eta^{\perp}\right\rangle<0, \text { or } \\
& \gamma_{1}<\gamma_{\ell},\left\langle h^{f}, \eta^{\perp}\right\rangle>0,\left\langle h^{g}, \eta^{\perp}\right\rangle>0 .
\end{aligned}
$$

Conditions (33)-(34) indicate that, depending on the balance between the degradation rates $\gamma_{1}$ and $\gamma_{\ell}$, there are two different settings that can lead to oscillations. Later in Section 5.3 we will examine how Assumption 2 constrains the class of transcriptional feedback that can induce oscillations.

It should be also pointed out that if Assumption 2 is not satisfied then there are three possibilities: there is stable sliding motion (or no sliding motion), trajectories never cross the switching boundary (in case both crossing domains are empty), or trajectories can cross only in one direction (in case one crossing domain is nonempty). From the results in the previous section, in all these cases a periodic orbit is not possible and trajectories will converge to a stable equilibrium.

From Figure 6 we observe that if the focal point $\phi_{1}^{f}$ lies outside its regular domain (i.e. $\phi^{f} \in D_{g}$ ), then $\phi_{2}^{f}<\beta \phi_{1}^{f}$ and substituting the intersection point $C_{f} \cap S=\left(x_{1}^{f}, \beta x_{1}^{f}\right)$ in the equation for $C_{f}(25)$ we get

$$
0=-\gamma_{1} \beta\left(x_{1}^{f}-\phi_{1}^{f}\right)+\gamma_{\ell}\left(\beta x_{1}^{f}-\phi_{2}^{f}\right)>\beta\left(\gamma_{1}-\gamma_{\ell}\right)\left(x_{1}^{f}-\phi_{1}^{f}\right)
$$

and therefore when $\gamma_{1}>\gamma_{\ell}$ we always have that $x_{1}^{f}>\phi_{1}^{f}$. A similar argument shows that $\phi^{g} \in D_{f}$ and $\gamma_{1}>\gamma_{\ell}$ imply $x_{1}^{g}<\phi_{1}^{g}$. We thus define the line segments

$$
\begin{aligned}
& S^{f}=\left\{x \in S: x_{1} \in\left[\phi_{1}^{f}, x_{1}^{f}\right]\right\}, \\
& S^{g}=\left\{x \in S: x_{1} \in\left[x_{1}^{g}, \phi_{1}^{g}\right]\right\},
\end{aligned}
$$

and their projections onto the $x_{1}$ axis

$$
\begin{aligned}
& S_{1}^{f}=\left\{x \in \mathbb{R}: x_{1} \in\left[\phi_{1}^{f}, x_{1}^{f}\right]\right\}, \\
& S_{1}^{g}=\left\{x \in \mathbb{R}: x_{1} \in\left[x_{1}^{g}, \phi_{1}^{g}\right]\right\},
\end{aligned}
$$

The segments $S_{1}^{f}$ and $S_{1}^{g}$ are shown in Figure 6; their definition is valid when $\gamma_{1}>\gamma_{\ell}$, but analogous versions can be defined in the converse case $\gamma_{1}<\gamma_{\ell}$ (the only difference is that their limits need to be reversed). The region $x_{1} \in\left[\phi_{1}^{f}, \phi_{1}^{g}\right]$, shown as shaded area in Figure 6 , is an invariant set since at $x_{1}<\phi_{1}^{f}$ (resp., $x_{1}>\phi_{1}^{g}$ ) one always has $\dot{x}_{1}>0$ (resp., $\dot{x}_{1}<0$ ). Periodic solutions can therefore cross the switching domain only in the segments $S^{f}$ and $S^{g}$. This means that trajectories starting on $x^{0} \in S^{f}$ will evolve in $D_{g}$ until they hit the segment $S^{g}$, re-enter $D_{f}$ and then return to the segment $S^{f}$. By constructing the Poincaré map from $S_{f}$ onto itself, we can prove the next result (details in Appendix B.3). 


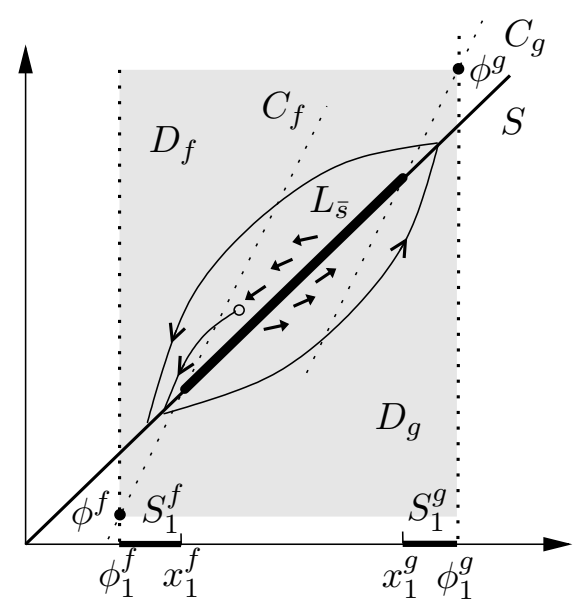

Figure 6: Sample trajectory for the case in Figure 4D with $\gamma_{1}>\gamma_{\ell}$; the shaded region is invariant under the PA dynamics.

Theorem 3 (Stable periodic orbit) Under Assumption 2 and with the focal points satisfying $\phi^{f} \in D_{g}$, $\phi^{g} \in D_{f}$, the PA system has a unique stable limit cycle.

In all the previous results we have assumed that the region for sliding motion is a line segment, i.e. $L_{s}$ and $L_{\bar{s}}$ are not isolated points. When the lines $C_{f}$ and $C_{g}$ coincide, these segments collapse to the point $L_{s}=L_{\bar{s}}=\phi_{s}$. Recalling (27), $C_{f}$ and $C_{g}$ match when $\left\langle h^{f}-h^{g}, \eta^{\perp}\right\rangle=0$, which is equivalent to

$$
\vartheta_{1}= \pm \frac{\pi}{2}
$$

This is a somewhat special case that is not covered by Theorems 1-3. If at least one of the focal points lies in its regular domain, then the solutions behave as described in Sections 4.3.1-4.3.2. On the contrary, if both focal points lie outside their regular domains, they will reach a singular equilibrium. This is stated in the next result, which is a consequence of Theorem 3.

Corollary 1 (No sliding motion) If $\phi^{f} \in D_{g}, \phi^{g} \in D_{f}$ and the angle $\vartheta_{1}= \pm \frac{\pi}{2}$, then the point $\phi_{s}$ in (30) is a stable singular equilibrium of the PA system.

\section{Detection of equilibria and oscillations}

In the case of different regulatory thresholds $\left(\theta_{1} \neq \theta_{\ell}\right)$, the metabolic-genetic circuit can be reduced to a PA system defined in three cones (Section 3). Although this scenario is more complex than the 2-cone case, it can be analyzed by splitting the 3 -cone problem into a pair of 2-cone ones. In the notation of Section 4, this approach translates into a 2-cone problem with:

$$
S=S_{1} \text { and focal points } \phi^{f}=\phi^{1}, \phi^{g}=\phi^{1 \ell},
$$

and another one with:

$$
S=S_{\ell} \text { and focal points } \phi^{f}=\phi^{1 \ell}, \phi^{g}=\phi^{\ell} .
$$

The location of the focal points $\left(\phi^{1}, \phi^{1 \ell}, \phi^{\ell}\right)$ depends on the regulatory logic and the protein degradation rates. However, from the different cases in Figure 2 we see that, regardless of the feedback logic and protein degradation, one pair of focal points will share the vertical coordinate and another pair will share 
the horizontal coordinate. With this simple observation and the two angle conditions in Theorems 12 we can systematically detect all possible equilibria for any configuration of repression and activation feedback loops. In what follows we illustrate our approach: in Sections 5.1-5.2 we study two cases that exhibit monostability and bistability, respectively, whereas in Section 5.3 we examine which regulatory configurations can generate metabolic oscillations.

\section{$5.1 \quad$ Single stable steady state}

Consider the case $\sigma_{1}=\sigma^{-}$and $\sigma_{\ell}=\sigma^{+}, \theta_{1}<\theta_{\ell}$. We will show that this configuration has a single steady state regardless of the location of the focal points. The fact that the pair $\left(\phi^{1}, \phi^{1 \ell}\right)$ share the horizontal coordinate, and $\left(\phi^{1 \ell}, \phi^{\ell}\right)$ share the vertical one (see Figure $2 \mathrm{C}$ ), limits the possible configurations of equilibria. There are five possible configurations:

(a) $\phi^{1} \in R_{1}, \phi^{1 \ell} \notin R_{1 \ell}$ and $\phi^{\ell} \notin R_{\ell}$;

(b) $\phi^{1 \ell} \in R_{1 \ell}, \phi^{1} \notin R_{1}$ and $\phi^{\ell} \notin R_{\ell}$;

(c) $\phi^{\ell} \in R_{\ell}, \phi^{1} \notin R_{1}$ and $\phi^{1 \ell} \notin R_{1 \ell}$;

(d) $\phi^{1 \ell} \in R_{1}, \phi^{1} \notin R_{1}$ and $\phi^{\ell} \in R_{1}$;

(e) $\phi^{1 \ell} \in R_{\ell}, \phi^{\ell} \notin R_{\ell}$ and $\phi^{1} \in R_{\ell}$.

In cases (a)-(c), the focal point that lies in its own regular domain (that is, $\phi^{1}, \phi^{1 \ell}$ and $\phi^{\ell}$ for cases (a), (b) and (c), respectively) is the only locally stable equilibrium (see discussion in Section 4.3.1). This equilibrium is also globally stable because, from any initial condition in $\mathbb{R}_{>0}^{2}$, trajectories will eventually enter the cone that contains its own focal point, from where they cannot escape because each coordinate is strictly monotone.

In configuration (d) no focal point belongs to its own regular domain, and therefore the only option is to look for singular equilibria in $S_{1}$ or $S_{\ell}$. Denote the normal vectors to $S_{1}$ and $S_{\ell}$ as $\eta_{1}^{\perp}$ and $\eta_{\ell}^{\perp}$, respectively. For the 2 -cone problem defined by $S_{1}$ we note that focal points $\phi^{f}=\phi^{1}$ and $\phi^{g}=\phi^{1 \ell}$ are aligned horizontally; we also have that

$$
\angle\left(h^{1}-h^{1 \ell}, \eta_{1}^{\perp}\right) \in\left[-\pi,-\frac{\pi}{2}\right)
$$

and therefore the angle condition (28) in Theorem 1 indicates that $S_{1}$ can contain a region with stable sliding motion. If we define $L_{\phi}$ as the line segment containing $\phi^{1}$ and $\phi^{1 \ell}$, then from Theorem 2 we conclude that the point

$$
\phi_{s}^{1}=L_{\phi} \cap S_{1}
$$

is a singular equilibrium; it is stable because the angle condition (31) is satisfied:

$$
\angle\left(\phi^{1}-\phi^{1 \ell}, \eta_{1}^{\perp}\right) \in\left[-\pi,-\frac{\pi}{2}\right) .
$$

For the pair of cones defined by $S_{\ell}$, the focal points $\phi^{f}=\phi^{1 \ell}$ and $\phi^{g}=\phi^{\ell}$ are aligned vertically. Moreover

$$
\angle\left(h^{1 \ell}-h^{\ell}, \eta_{\ell}^{\perp}\right) \in\left(\frac{\pi}{2}, \pi\right]
$$

which by Theorem 1 implies that $S_{\ell}$ also contains a region for sliding motion. However, in this case the line segment containing $\phi^{1 \ell}$ and $\phi^{\ell}$ does not intersect the switching domain $S_{\ell}$. Therefore, by Theorem 
$2, S_{\ell}$ does not contain a singular equilibrium. We therefore conclude that $\phi_{s}^{1}$ is the only equilibrium of the system. Configuration (e) can be analyzed similarly to show that it can only have one stable singular equilibrium in $S_{\ell}$.

Simulated trajectories of configuration (d) are shown in Figure 7 for a system with $n=2$ metabolites and regulation from the product $\left(s_{\ell-1}=s_{2}\right)$. We have deliberately used enzyme kinetics that are faster than protein degradation. To validate the effectivity of the timescale separation, the protein trajectories of the PA system (blue) are shown together with those of the original system in (1)-(2) (without the QSS approximation, in green). We are able to predict the equilibrium of both the original system and its PA reduction, located at $\phi_{s}^{1}$ (marked with a square in Figure 7).
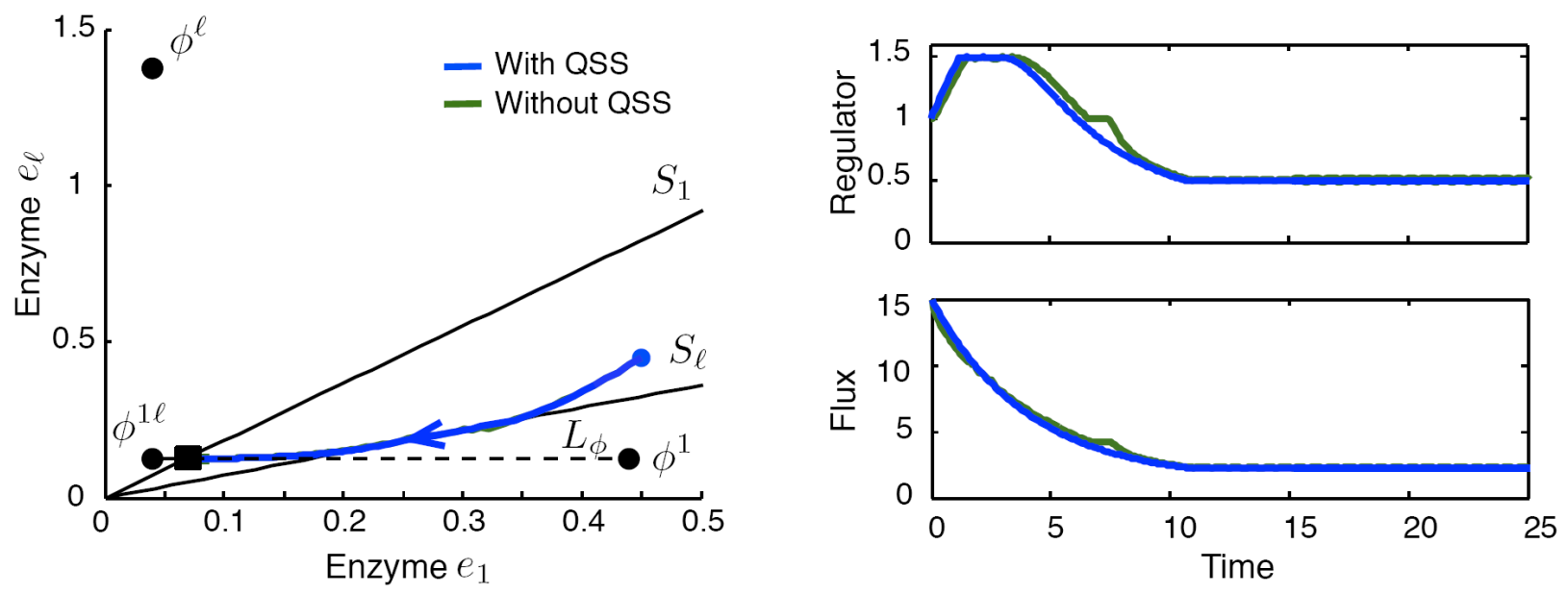

Figure 7: Enzyme phase plane, trajectory of the regulator $\left(s_{2}(t)\right)$, and resulting flux $\left(v_{3}(t)\right)$ for a monostable system with regulation $\left(\sigma_{1}=\sigma^{-}, \sigma_{\ell}=\sigma^{+}\right)$. The substrate is $s_{0}=1$, protein degradation rates $\gamma_{i}=$ $\{0.25,0.9,0.8\}$, and the enzyme kinetics are of Michaelis-Menten type $\left(g_{i}=k_{\text {cat } i} s_{i-1} /\left(K_{\mathrm{m} i}+s_{i-1}\right)\right)$ with parameters chosen so that metabolic reactions are much faster than protein expression, $k_{\text {cat } i}=2 \cdot 10^{2}$ and $K_{\mathrm{m} i}=5, i=1,2,3$. The regulatory parameters are $\theta_{i}=\{0.5,1,1.5\}, \kappa_{i}^{0}=\{0.01,0.15,0.1\}$, and $\kappa_{i}^{1}=10 \kappa_{i}^{0}$.

\subsection{Bistability}

Consider the case of positive regulation $\left(\sigma_{1}=\sigma^{+}\right.$and $\left.\sigma_{\ell}=\sigma^{+}\right)$with $\theta_{1}<\theta_{\ell}$, and the focal points located as in Figure 8. In the 2-cone problem for $R^{1}$ and $R^{1 \ell}$, we note that $\phi^{f}=\phi^{1} \in R_{1}$ and $\phi^{g}=\phi^{1 \ell} \notin R_{1} \cup R_{1 \ell}$, and therefore $\phi^{1}$ is the only locally stable equilibrium. In the 2 -cone problem for $R^{1 \ell}$ and $R^{\ell}$, both $\phi^{f}=\phi^{1 \ell}$ and $\phi^{g}=\phi^{\ell}$ lie outside their regular domains, and they are aligned vertically. The angle condition (28):

$$
-\pi \leq \angle\left(h^{1 \ell}-h^{\ell}, \eta_{\ell}^{\perp}\right) \leq-\frac{\pi}{2}
$$

holds, and so by Theorem 1 the switching domain $S_{1}$ can contain a region with stable sliding motion. If we define $L_{\phi}$ as the line segment containing $\phi^{1 \ell}$ and $\phi^{\ell}$, then from Theorem 2 we conclude that the point

$$
\phi_{s}^{\ell}=L_{\phi} \cap S_{\ell}
$$

is a singular equilibrium; it is stable because the angle condition (31) is satisfied:

$$
\angle\left(\phi^{1 \ell}-\phi^{\ell}, \eta_{\ell}^{\perp}\right) \in\left[-\pi,-\frac{\pi}{2}\right)
$$


We therefore conclude that the system has two locally stable equilibria located at $\phi^{1}$ and $\phi_{s}^{\ell}$. The simulation results in Figure 8 verify our predictions, showing two sample trajectories that converge to the different equilibrium points (marked with squares).
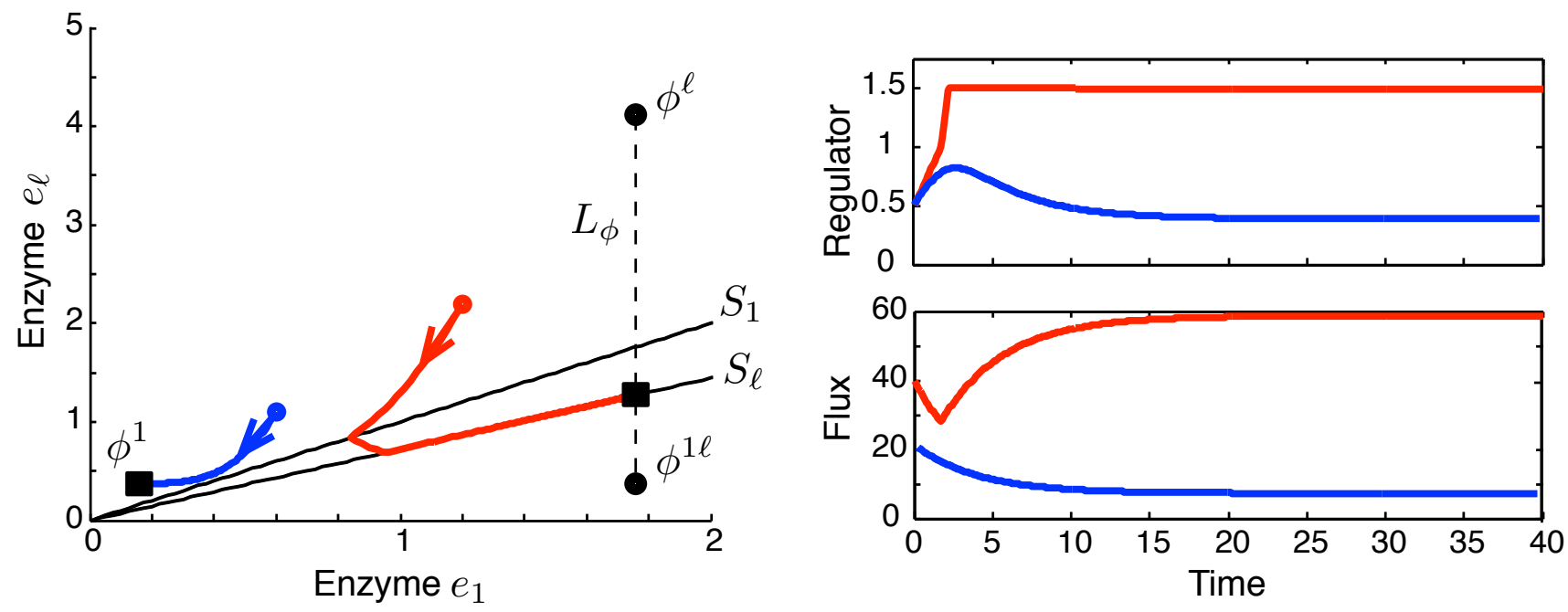

Figure 8: Enzyme phase plane, trajectory of the regulator $\left(s_{2}(t)\right)$, and resulting flux $\left(v_{3}(t)\right)$ for a bistable system with positive regulation $\left(\sigma_{1}=\sigma^{+}, \sigma_{\ell}=\sigma^{+}\right)$. The regulatory parameters are $\theta_{i}=\{1,0.75,1.5\}$, $\kappa_{i}^{0}=\{0.04,0.3,0.3\}, \kappa_{i}^{1}=\{0.4,2,3\}$. All the remaining model parameters are identical to those used in Figure 7.

\subsection{Oscillations}

A special feature of the 3-cone problem is that its focal points are pairwise aligned vertically or horizontally (see Figure 2). In view of the analysis in Section 4.4, two of the necessary conditions for oscillations in the 2-cone system are that: (i) there exists unstable sliding motion in the switching domain, and (ii) the focal points are located outside their regular domains. Once we split the 3-cone problem into a pair 2-cone ones, from Figure 3 we see that if (ii) is satisfied and a pair of focal points are aligned vertically or horizontally, the the angle $\vartheta_{1} \in[-\pi,-\pi / 2] \cup[\pi / 2, \pi]$ and so (i) cannot be satisfied because $L_{\bar{s}}=\emptyset$ (by Theorem 1 ).

The above discussion indicates that because the focal points in the 3 -cone case are geometrically constrained, conditions (i)-(ii) cannot be simultaneously satisfied in any of the feedback configurations of Figure 2. However, under equal regulatory thresholds $\left(\theta_{1}=\theta_{\ell}\right)$ the 3 -cone problem reduces a 2-cone one with focal points $\left(\phi^{1}\right.$ and $\left.\phi^{\ell}\right)$ that can be located anywhere in the positive quadrant, and hence oscillations are possible. The regulatory logic plays in critical role on the location of these two focal points. This is reflected in the next result, which identifies which regulatory configurations can lead to metabolic oscillations for different balances between enzyme degradation rates.

Corollary 2 (Metabolic oscillations) Consider the 3-cone system in (17) with $\theta_{1}=\theta_{\ell}$ and the focal points outside their regular domains $\left(\phi^{1} \in R_{\ell}\right.$ and $\left.\phi^{\ell} \in R_{1}\right)$. The system exhibits a unique stable limit cycle if:

A. $\sigma_{1}=\sigma^{-}, \sigma_{\ell}=\sigma^{-}, \gamma_{1}<\gamma_{\ell}$, or

B. $\sigma_{1}=\sigma^{+}, \sigma_{\ell}=\sigma^{+}, \gamma_{1}>\gamma_{\ell}$. 
The proof of the above result has been omitted for brevity, but it follows directly from the conditions in Section 4.4. In terms of regulatory logic, Corollary 2 indicates that there are only two scenarios where oscillations can appear (these are the ones shown in Figures $2 \mathrm{~A}$ and 2D), and mixed logic configurations (shown Figures 2B and 2C) do not allow for oscillatory behavior. In Figure 9 we show two sample oscillatory responses of a system with $n=2$ metabolites and negative regulation from the product $\left(s_{\ell-1}=s_{2}\right)$.
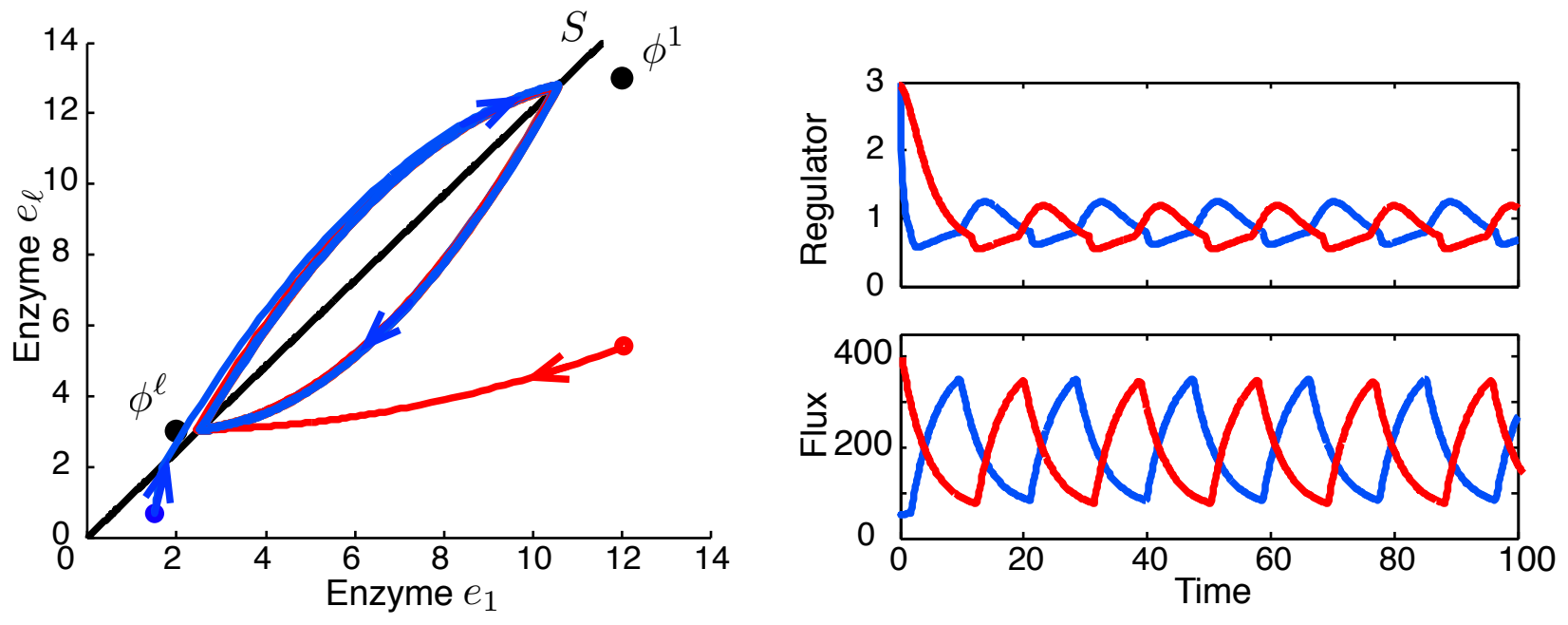

Figure 9: Enzyme phase plane, trajectory of the regulator $\left(s_{2}(t)\right)$, and resulting flux $\left(v_{3}(t)\right)$ for an oscillatory system with negative regulation $\left(\sigma_{1}=\sigma^{-}, \sigma_{\ell}=\sigma^{-}\right)$. The regulatory parameters are $\theta_{i}=\{0.8,1,0.8\}$, $\kappa_{i}^{0}=\{0.5,1.5,1.5\}, \kappa_{i}^{1}=\{2.5,1.5,5\}$, and degradation rates $\gamma_{i}=\{0.25,0.8,0.5\}$. All the remaining model parameters are identical to those used in Figure 7.

\section{Regulation via an operon: substrate-induced bifurcations}

A common regulatory structure in bacteria are gene operons, whereby several genes are controlled by the same promoter and thus transcribed in response to the same activity threshold (examples are the lac and trp operons $[9,10])$. The previous case studies (Section 5) demonstrate that the ordering of the activity thresholds $\left(\theta_{1}, \theta_{\ell}\right)$ defines fundamental properties of the system dynamics. In particular, distinct threshold values always lead to (multi-)stability, whereas the case of equal thresholds introduces the possibility of oscillatory behavior.

In this section we focus on the behavior of metabolic networks controlled via an operon, and how this affects the bifurcation diagram of the metabolic flux as a function of the substrate. A widely studied instance of this type of regulatory structure is the lac operon, with a range of mathematical models developed in the literature (see $[8,9]$ and the references therein). For our purposes, a simplified description of the lac operon in the form of the generic model in (1)-(2) can be constructed by (see Figure 10) setting $s_{0}=$ lactose (extracellular), $s_{1}=$ lactose (internal), and $s_{2}=$ allolactose, with the enzymes defined by $e_{0}=$ permease (coded by $l a c Y$ ), and $e_{1}=\beta$-galactosidase (coded by lac $Z$ ). The regulator is $s_{2}$ (allolatose), which binds the transcription factor $(\mathrm{TF})$ and prevents it from blocking operon transcription, which in terms of our model translates into setting $\sigma_{i}=\sigma^{+}$with thresholds $\theta_{i}=\theta$ for $i=1,2,3$.

In the previous sections we have shown how to detect the equilibria and limit cycles of the regulatory system. We now use the strategy to determine the bifurcation diagram of the feedback system as a function 
of the external substrate. In the case of equal thresholds (Section 5.3), the slope of the only switching domain is

$$
\beta=\frac{g_{1}\left(s_{0}\right)}{g_{3}(\theta)} .
$$

The nutrient concentration scales the slope $\beta$ through turnover rate of the first enzyme $g_{1}\left(s_{0}\right)$. For a given combination of protein expression and degradation rates (i.e. fixed focal points), changes in the extracellular substrate can modify the relative location of the focal points and regular domains. This phenomenon therefore induces bifurcations in the dynamics as a function of the nutrient availability.

In Figure 11A we observe the case of nutrient-induced bistability for a bounded range of nutrient concentrations. In this scenario, the nutrient availability can drive the system between monostable and bistable regions. The monostable regions exhibit a metabolic flux that increases with the nutrient availability, and the bistable region can display low and high flux regimes. The bistable behavior in Figure 11A is consistent with experimental observations in E. coli populations [19]. In that work it was shown that under low (resp. high) external lactose concentration, the lac operon genes were expressed at low (resp. high) levels. For an intermediate range of external lactose, the response of the population was shown to be bimodal, as two types of cells were observed: those that do and those that do not transcribed the lac genes - without an "intermediate level transcription". (See Figures 2 and 4 in [19].)

The analysis in Section 5.3 showed that oscillatory behavior can appear under positive or negative regulation. As shown in Figure 11B, operon regulation can induce metabolic oscillations, but this depends on the balance between protein half lives (see the conditions in Corollary 2). Oscillatory flux may only appear when the half-life of the nutrient-uptake enzyme is shorter than that of the proteins that breakdown or consume it (blue line in Figure 11B). Otherwise the system exhibits a unique metabolic flux.

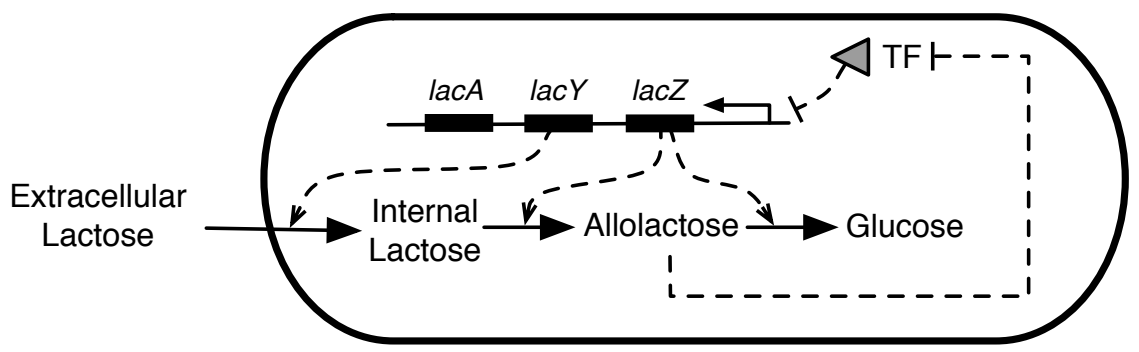

Figure 10: Representation of the lac operon in terms of the generic model in Figure 1A.

\section{Discussion}

In this paper we have investigated how genetic regulation of enzyme activity can generate different phenotypes in an unbranched metabolic network. The two key elements in our analysis are: (i) the use of a piecewise affine (PA) model for gene regulation, and (ii) the time scale separation between metabolism and gene expression. The PA model describes how gene expression is switched ON or OFF in response to a metabolite that acts as a global regulator, whereas the time scale separation allows the reduction of the PA model to a 2-dimensional system.

The chosen formalism allowed for a complete theoretical analysis of the mechanisms by which one-to-all gene regulatory circuits can generate different metabolic phenotypes. In the reduced model we found that only two enzymes are needed to characterize the system: the one catalyzing the first reaction step, and the one catalyzing the reaction that consumes the regulator. Since the regulator can either activate or repress 

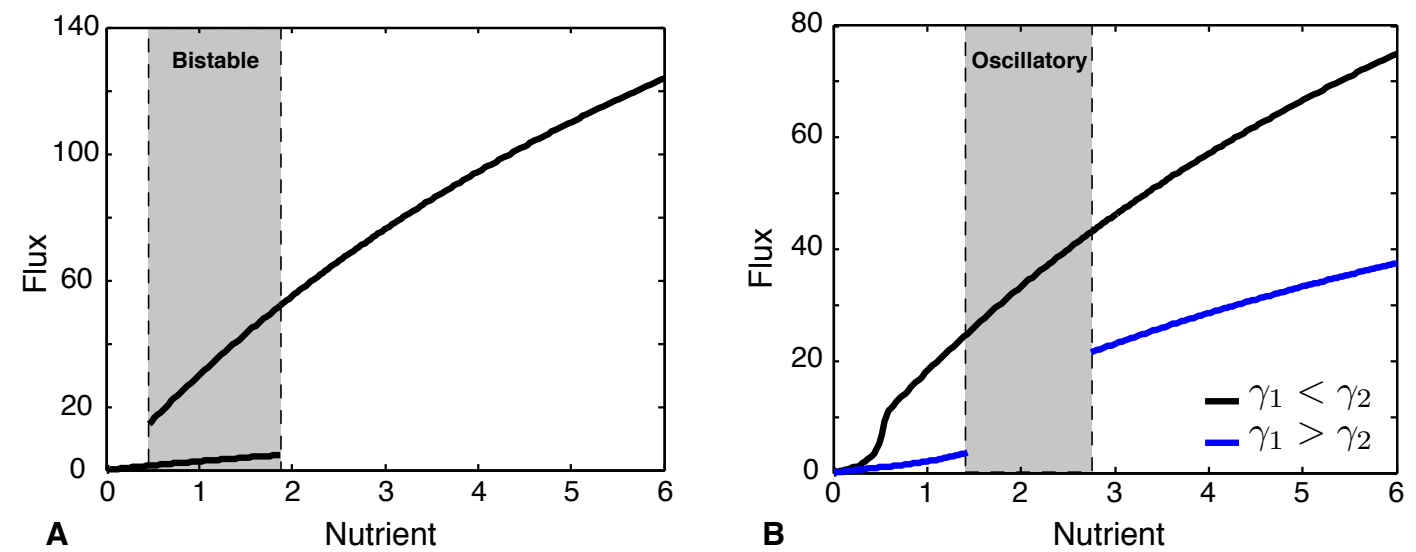

Figure 11: Bifurcation diagrams for operon-controlled unbranched metabolic network. (A) Positive regulation can lead to bistability; regulatory parameters are $\theta=0.5, \kappa_{i}^{0}=\{0.3,1\}, \kappa_{i}^{1}=\{3,2\}$, and equal protein degradation $\gamma_{i}=1, i=1,2$. (B) Positive regulation can also lead to oscillatory behavior, but this depends on the balance between protein half-lives; regulatory parameters are $\theta=0.5, \kappa_{i}^{0}=\{0.4,0.3\}$, $\kappa_{i}^{1}=\{1.6,4.2\}$; protein degradation rates are $\gamma_{i}=\{1,2\}$ (black line), and $\gamma_{i}=\{2,1\}$ (blue line), respectively. Both bifurcation diagrams were generated using the results for PA systems for a network with one metabolite and $n=2$ enzymes. The enzyme kinetics have been chosen much faster than protein half-lives (Michaelis-Menten kinetics with parameters $k_{\text {cat } i}=100$ and $K_{\mathrm{m} i}=10, i=1,2$.).

each enzyme, there are four different combinations of regulatory logic. For each of these configurations, the enzyme phase plane can be partitioned into three conic regions (see Figure 2). The location of the focal points relative to these regions determine the existence of stable equilibria and periodic oscillations in the complete system.

In general, we observe that multistable behavior can appear for specific combinations of enzyme expression rates and regulatory thresholds. Among the four possible feedback configurations, only one (namely, $\sigma_{1}=\sigma^{-}, \sigma_{\ell}=\sigma^{+}$) leads to the existence of a single steady state independently of the parameter values (see Section 5.1). All other combinations allow mono- or multistability for specific parameter values; in particular, the logic $\sigma_{1}=\sigma^{+}, \sigma_{\ell}=\sigma^{-}$can display up to three equilibria (see Figure 2B, whereby each of the three focal points belongs to its own regular domain). This observation suggests that a feedback logic of the form $\left(\sigma_{1}=\sigma^{-}, \sigma_{\ell}=\sigma^{+}\right)$is optimal to guarantee a unique phenotype that is robust to parameter values and environmental conditions (encoded in the substrate concentration $s_{0}$ ). Intuitively, the $\left(\sigma_{1}=\sigma^{-}, \sigma_{\ell}=\sigma^{+}\right)$logic causes the regulator to self-repress, in the sense that it blocks its own production and accelerates its transformation, and therefore leads to only one possible phenotype.

Our analysis also reveals fundamental differences between the regulation of gene expression through operons and individual genes. Individual gene regulation translates into a 3-cone partition of the enzyme state space, and therefore the metabolic flux can exhibit complex bifurcations as a function of the regulatory parameters (these in turn affect the location of the focal points). In contrast, an operon architecture translates into a 2-cone partition that displays a simpler bifurcation structure: parameters can change in broader regions without necessarily changing the location of the focal points with respect to the cones. As a consequence, an operon structure can provide sharp metabolic regulation by allowing specific phenotypes (mono-, multistability, or oscillatory behavior) to be conserved across a larger parameter range than individual gene regulation.

The conclusions drawn from our formalism exploit the switch-like form of the model for gene expression. Because of the discontinuous nature of this class of models, solutions may not depend continuously on model parameters (in contrast with classical continuous systems). As a consequence, our theory predicts 
that metabolic oscillations emerge only under identical thresholds. A continuous version of (1)-(2) can indeed display oscillations even when the thresholds are slightly different; this phenomenon was observed in simulations with Hill-type [16] regulatory functions $\sigma_{i}$ and high Hill coefficients (not shown here).

Our results also rely on the timescale separation between the metabolic and genetic subsystems. This assumption is based on the fact that metabolic reactions typically operate in the order of seconds or faster, whereas genetic adaptations last from minutes up to several hours. The reduced model will be a good approximation of the complete system provided that the time constants of the enzyme kinetics are much smaller than protein half-lives, which in turn are inversely proportional to their degradation rates. If we do not impose the QSS approximation, the downstream pathway dynamics introduce a lag between changes in enzyme concentrations and changes in metabolite trajectories. The enzyme trajectories may therefore display a small "inertia" effect at the switching boundaries between the cones. This inertia will be larger for those enzymes that are regulated by a metabolite far downstream the pathway, but it does not change our conclusions regarding the existence, location, and stability of equilibria.

The analysis presented in this paper contributes to understanding the interactions between genetic and metabolic networks. Unbranched networks under one-to-all transcriptional control (also called "single input modules") are a common building block of more complex metabolic systems [7]. We have shown that a hybrid formalism, which combines continuous metabolic dynamics with switch-like gene expression, can be effectively used to detect mechanisms that generate complex behavior in relatively simple motifs. In addition, the analysis provides novel insights into the design of metabolic-genetic systems for prescribed behaviors such as multistability and periodic oscillations.

\section{Acknowledgements}

This work was supported by the French research agency through project Gemco (ANR2010 BLANC020101), and an NBIPI Career Enhancement and Mobility Fellowship (co-funded by Marie Curie Actions, the Irish Higher Education Authority Programme for Third Level Institutions Cycle 4 and the Italian National Research Council).

\section{References}

[1] Oliver Kotte, Judith B Zaugg, and Matthias Heinemann. Bacterial adaptation through distributed sensing of metabolic fluxes. Mol Syst Biol, 6(355), 2010.

[2] Chen-Hsiang Yeang. Integration of metabolic reactions and gene regulation. Molecular Biotechnology, 47(1):70-82, August 2010.

[3] Eileen Fung, Wilson W. Wong, Jason K. Suen, Thomas Bulter, Sun gu Lee, and James C. Liao. A synthetic gene-metabolic oscillator. Nature, 435(7038):118-122, May 2005.

[4] K. Bettenbrock, S. Fischer, A. Kremling, K. Jahreis, T. Sauter, and E.D. Gilles. A quantitative approach to catabolite repression in Escherichia coli. Journal of Biological Chemistry, 281(5):2578$2584,2006$.

[5] Anne Goelzer, Fadia Bekkal Brikci, Isabelle Martin-Verstraete, Philippe Noirot, Philippe Bessieres, Stephane Aymerich, and Vincent Fromion. Reconstruction and analysis of the genetic and metabolic regulatory networks of the central metabolism of bacillus subtilis. BMC Systems Biology, 2(1):20, 2008. 
[6] Tomer Shlomi, Yariv Eisenberg, Roded Sharan, and Eytan Ruppin. A genome-scale computational study of the interplay between transcriptional regulation and metabolism. Mol Syst Biol, 3, April 2007.

[7] Shai S. Shen-Orr, Ron Milo, Shmoolik Mangan, and Uri Alon. Network motifs in the transcriptional regulation network of escherichia coli. Nat Genet, 31(1):64-68, May 2002.

[8] Necmettin Yildirim and Michael C. Mackey. Feedback regulation in the lactose operon: A mathematical modeling study and comparison with experimental data. Biophysical Journal, 84(5):2841-2851, May 2003.

[9] P. Wong, S. Gladney, and J.D. Keasling. Mathematical model of the lac operon: Inducer exclusion, catabolite repression, and diauxic growth on glucose and lactose. Biotechnology Progress, 13(2):132$143,1997$.

[10] Moisés Santillán and Michael C. Mackey. Dynamic regulation of the tryptophan operon: A modeling study and comparison with experimental data. Proceedings of the National Academy of Sciences of the United States of America, 98(4):1364-1369, February 2001. PMID: 11171956 PMCID: 29262.

[11] A. Zaslaver, A. Mayo, R. Rosenberg, P. Bashkin, H. Sberro, M. Tsalyuk, M. Surette, and U. Alon. Just-in-time transcription program in metabolic pathways. Nature Genetics, 36(5):486-491, May 2004.

[12] Leon Glass and Stuart A. Kauffman. The logical analysis of continuous, nonlinear biochemical control networks. J. Theor. Biol., 39:103-129, 1973.

[13] R. Edwards. Analysis of continuous-time switching networks. Pysica D: Nonlinear Phenomena, 146:165-199, 2000.

[14] Jean-Luc Gouzé and Tewfik Sari. A class of piecewise linear differential equations arising in biological models. Dynamical Systems, 17(4):299-316, 2002.

[15] Richard Casey, Hidde de Jong, and Jean-Luc Gouzé. Piecewise-linear models of genetic regulatory networks: equilibria and their stability. J. Math. Biol., 52:27-56, 2006.

[16] Siren R Veflingstad and Erik Plahte. Analysis of gene regulatory network models with graded and binary transcriptional responses. Bio Systems, 90(2):323-339, October 2007. PMID: 17118528.

[17] Hidde de Jong, Jean-Luc Gouzé, Céline Hernandez, Michel Page, Tewfik Sari, and Johannes Geiselmann. Qualitative simulation of genetic regulatory networks using piecewise-linear models. Bulletin of Mathematical Biology, 2:301-340, 2004.

[18] Aleksei F. Filippov. Differential equations with discontinuous righthand-sides. Kluwer Academics Publishers, 1988.

[19] E.M. Ozbudak, M. Thattai, H.N. Lim, B.L. Shraiman, and A. van Oudenaarden. Multistability in the lactose utilization network of Escherichia coli. Nature, 427:737-740, 2004.

[20] D. Ropers, H. de Jong, M. Page, D. Schneider, and J. Geiselmann. Qualitative simulation of the carbon starvation response in Escherichia coli. BioSystems, 84:124-152, 2006.

[21] A. Cornish-Bowden. Fundamentals of Enzyme Kinetics. Portland Press, third edition, 2004.

[22] Uri Alon. An introduction to systems biology: design principles of biological circuits. Chapman \& Hall/CRC, 2006. 
[23] L.C.G.J.M. Habets and Jan van Schuppen. A control problem for affine dynamical systems on a full-dimensional polytope. Automatica, 40:21-35, 2004.

[24] D. A. Oyarzún and M. Chaves. Global gene regulation in metabolic networks. In Proc. $18^{\text {th }}$ IFAC World Congress, Milan, Italy, August 2011.

[25] F. Giannakopoulos and K. Pliete. Planar systems of piecewise differential equations with a line of discontinuity. Nonlinearity, 14(6):1611-1632, 2001.

\section{Appendix}

\section{A Valid equilibria and quasi steady state approximation}

Existence of metabolic equilibria If the enzymatic and metabolic equilibria are denoted as $\bar{e}_{i}$ and $\bar{s}_{i}$ respectively, then the mass balance model (1) under Assumption 1 leads to

$$
g_{i+1}\left(\bar{s}_{i}\right) \bar{e}_{i+1}=g_{1}\left(s_{0}\right) \bar{e}_{1}
$$

for $i=1,2, \ldots, n$. The above is an implicit equation for the equilibrium $\bar{s}_{i}$ as a function of the enzymatic equilibria. The existence of a valid (i.e. nonnegative) solution of (49) depends on the saturation value of the enzyme kinetics, denoted as $\hat{g}_{i}=\max g_{i}\left(s_{i-1}\right)$. For given enzymatic equilibrium, a nonnegative solution of (49) exists only when

$$
\bar{e}_{i} \geq \frac{g_{1}\left(s_{0}\right)}{\hat{g}_{i}} \bar{e}_{1}
$$

for each $i=2,3, \ldots, n$. Geometrically, condition (50) means that the enzyme equilibrium vector must belong to the $(n+1)$-dimensional cone

$$
\hat{R}=\left\{x \in \mathbb{R}_{\geq 0}^{n+1}: x_{i} \geq \frac{g_{1}\left(s_{0}\right)}{\hat{g}_{i}} x_{1}, i \geq 2\right\} .
$$

Since we do not know a priori the location of the enzymatic equilibria, we cannot give tight conditions under which (50) holds. However, we can obtain bounds for the parameter space by looking at the limit case of (50). In a worst-case scenario, the OFF levels $E_{i}^{\text {off }}, i \geq 2$, must satisfy (50) against the ON level of the first enzyme, $E_{1}^{\text {on }}$. This condition becomes

$$
E_{i}^{\text {off }} \geq \frac{g_{1}\left(s_{0}\right)}{\hat{g}_{i}} E_{1}^{\text {on }}
$$

for $i=2,3, \ldots n+1$, and provides bounds (albeit conservative) for the synthesis and degradation rates that ensure the existence of a metabolic equilibrium. As a consequence of the feedback interaction between the genetic and metabolic subsystems, the bounds in (52) relate purely genetic parameters with metabolic properties such as enzyme kinetics and substrate concentration.

Solvability of the quasi steady state approximation For the same reasons discussed above, a positive solution $s_{i-1}(t)$ of (9) exists only when

$$
e(t) \in \hat{R}, \text { for all } t \geq 0 .
$$


The cone $\hat{R}$ therefore defines a region in the enzyme state space that guarantees the solvability of the QSS approximation. This constraint is stronger than condition (50), which is required to hold in equilibrium only. Nevertheless, if the protein trajectories start in $\hat{R}$ (i.e. $e(0) \in \hat{R}$ ), as $s_{i-1}$ grows the pair $\left(e_{1}, e_{i}\right)$ in (9) will approach the boundary of $\hat{R}$, but by continuity they cannot leave $\hat{R}$. Moreover, $e(0) \in \hat{R}$ can be ensured by picking any initial equilibrium flux $V=v_{i}(0)$ for all $i$, together with consistent initial enzyme concentrations:

$$
e_{i}(0)=\frac{V}{g_{i}\left(s_{i-1}(0)\right)}=\frac{g_{1}\left(s_{0}\right)}{g_{i}\left(s_{i-1}(0)\right)} e_{1}(0)
$$

Since $g_{i}\left(s_{i-1}(0)\right)<\hat{g}_{i}$ for any finite $s_{i-1}(0)$, we see that initial enzyme concentrations that are consistent with an equilibrium flux are enough to guarantee that $e(0) \in \hat{R}$ and therefore ensure solvability of the QSS approximation.

\section{B Piecewise affine systems in cones}

\section{B.1 Proof of Theorem 1.}

Solutions of differential equations with discontinuous vector fields are typically characterized with a construction due to Filippov [18]. This method proceeds by extending (23) to a differential inclusion

$$
\dot{x} \in H(x), \forall x \in S,
$$

where $H(x)$ is a set-valued function defined as the closed convex hull of $f(x)$ and $g(x)$, i.e.

$$
H(x)=\left\{z \in \mathbb{R}^{2}: z=\alpha f(x)+(1-\alpha) g(x), 0 \leq \alpha \leq 1\right\} .
$$

The solutions of (55) are understood in the following sense (see also [14, 15] for more details).

Definition 1 For a given $\rho_{0}$, a solution of $(55)$ in $[0, T]$ is an absolutely continuous function $\rho:[0, T] \rightarrow$ $\mathbb{R}_{\geq 0}^{2}$ such that $\rho(0)=\rho_{0}$ and $\dot{\rho}(t) \in H(\rho(t))$ for almost all $t \in[0, T]$.

Depending on the directions of the vector fields $f(x)$ and $g(x)$, Filippov's construction may not allow for uniqueness of solutions in the switching domains [18]. When uniqueness can be guaranteed, then solutions can: (a) cross to a regular domain or, (b) slide along the switching surface $S$. Roughly speaking, case (a) occurs when $f(x)$ and $g(x)$ point in similar directions in a vicinity of $S$, so that the vectors in $H(x)$ point toward a regular domain irrespective of $\alpha$. In case (b) both vector fields point towards the switching domain, so that one can find a unique value of $\alpha$ such that $H(x)$ points in the direction of $S$ (in this case we say that the solution exhibits stable sliding motion in $S$ ). Uniqueness of solutions is lost when both vector fields point away from the switching domain, in which case solutions starting at $S$ cannot be uniquely defined and any small perturbation will drive $x$ away from $S$ (referred to as an unstable sliding motion).

Part (a) From Filippov's construction, the vector field in $S$ has the form

$$
\dot{x}=\left[\begin{array}{l}
\alpha f_{1}(x)+(1-\alpha) g_{1}(x) \\
\alpha f_{2}(x)+(1-\alpha) g_{2}(x)
\end{array}\right]
$$

with $0 \leq \alpha \leq 1$. By the definition of $\Omega_{f}^{+}$and $\Omega_{g}^{+}$, for any point $x \in L_{g f}$ we have that

$$
\begin{aligned}
\alpha f_{2}(x)+(1-\alpha) g_{2}(x) & >\alpha \beta f_{1}(x)+(1-\alpha) \beta g_{1}(x), \\
& >\beta\left(\alpha f_{1}(x)+(1-\alpha) g_{1}(x)\right),
\end{aligned}
$$

which implies that the vector field points to $D_{f}$ for any $0 \leq \alpha \leq 1$. In other words, the set $H(x)$ in $(55)$ is fully contained in the regular domain $D_{f}$ and hence the trajectory crosses the switching domain. 
Part (b) Analogous to part (a).

Part (c) We first prove by contradiction that for all $x\left(t_{0}\right) \in L_{s}$ the vector fields are such that $x(t)$ cannot leave the switching domain in an interval $\left(t_{0}, t_{0}+\Delta\right]$. Define the absolutely continuous function

$$
\begin{array}{r}
z:\left[t_{0}, t_{0}+\Delta\right] \rightarrow \mathbb{R}, \\
z(t)=x_{2}(t)-\beta x_{1}(t) .
\end{array}
$$

Suppose that there exists $\Delta>0$ such that $z(t)>0$ for $t \in\left(t_{0}, t_{0}+\Delta\right]$. If $x\left(t_{0}\right) \in S$ we have that $z\left(t_{0}\right)=0$, so by continuity it must be that $\dot{z}>0$ for $t \in\left(t_{0}, t_{0}+\Delta_{0}\right]$ and some $0<\Delta_{0} \leq \Delta$. In addition, from the definition of the PA system in (23), if $z(t)>0$ then $\dot{x}=f(x)$ for $t \in\left(t_{0}, t_{0}+\Delta_{0}\right]$ and so

$$
\dot{z}=f_{2}(x)-\beta f_{1}(x) \text {, for } t \in\left(t_{0}, t_{0}+\Delta_{0}\right] .
$$

However, the right-hand side of (59) is continuous in $t$, and when $x\left(t_{0}\right) \in L_{s}$ it follows that $\dot{z} \leq 0$ for $t \in\left(t_{0}, t_{0}+\Delta_{0}\right]$, which is a contradiction. The converse argument can be used to show that $z(t)<0$ for $t \in\left(t_{0}, t_{0}+\Delta\right]$ leads to a contradiction. We thus conclude that $z(t)=0$ for $t \in\left[t_{0}, t_{0}+\Delta\right]$, and so $x(t) \in L_{s}$ for $t \in\left[t_{0}, t_{0}+\Delta\right]$.

The proof follows by checking that the vector fields for $x \in L_{s}$ are compatible with Filippov's construction, see [14]. If there is sliding motion in $L_{s}$, then there exists $\Delta>0$ such that

$$
\dot{z}=0, \text { for } t \in\left[t_{0}, t_{0}+\Delta\right] \text {. }
$$

Since $x(t)$ must be a solution in Filippov's sense for $t \in\left[t_{0}, t_{0}+\Delta\right]$, then there must exist $0 \leq \alpha \leq 1$ such that

$$
\dot{x}=\alpha f(x)+(1-\alpha) g(x), \text { for } t \in\left[t_{0}, t_{0}+\Delta\right]
$$

Combining (60) and (61) we get

$$
\begin{aligned}
0 & =\dot{x}_{2}-\beta \dot{x}_{1}, \\
& =\alpha f_{2}+(1-\alpha) g_{2}-\beta\left(\alpha f_{1}+(1-\alpha) g_{1}\right), \\
& =\alpha\left(f_{2}-\beta f_{1}\right)+(1-\alpha)\left(g_{2}-\beta g_{1}\right), \text { for } t \in\left[t_{0}, t_{0}+\Delta\right] .
\end{aligned}
$$

Solving for $\alpha$ in (62) gives

$$
\alpha(x)=\frac{g_{2}(x)-\beta g_{1}(x)}{\left(g_{2}(x)-\beta g_{1}(x)\right)-\left(f_{2}(x)-\beta f_{1}(x)\right)},
$$

For $x \in L_{s}$ it holds that $\left(f_{2}-\beta f_{1}\right) \leq 0$ and $\left(g_{2}-\beta g_{1}\right)>0$, therefore $\alpha(x)$ is unique for all $x \in L_{s}$ and satisfies $0 \leq \alpha(x) \leq 1$.

Part (d) Consider the function $z(t)$ defined in (58). As opposed to the proof of part (c), in this case it can be shown that for $x\left(t_{0}\right) \in L_{\bar{s}}$ both $z(t)>0$ and $z(t)<0$ for $t \in\left(t_{0}, t_{0}+\Delta\right]$ are possible solutions. Note that another possible solution can be defined by picking $\alpha$ as in (63) so that $x(t)$ slides along $S$.

The angle conditions in (28)-(29) can be obtained as follows. The lines $C_{f}$ and $C_{g}$ intercept the vertical axis of $\mathbb{R}_{\geq 0}^{2}$ at $p^{f}=\gamma_{\ell}^{-1}\left\langle h^{f}, \eta^{\perp}\right\rangle$ and $p^{g}=\gamma_{\ell}^{-1}\left\langle h^{g}, \eta^{\perp}\right\rangle$, respectively. From Figure 3 we see that whether $L_{s}=\emptyset$ or $L_{\bar{s}}=\emptyset$ depends on $\operatorname{sgn}\left(p^{f}-p^{g}\right)=\operatorname{sgn}\left\langle h^{f}-h^{g}, \eta^{\perp}\right\rangle$, which leads to the conditions in (28)-(29). 


\section{B.2 Proof of Theorem 2.}

A singular equilibrium must be understood in Filippov's sense, i.e. at a singular equilibrium the convex hull $H(x)$ in (55) contains the origin. The proof follows by looking at the form of the vector field along $S$ when solutions are defined with Filippov's method. When $x \in L_{s}$ the solution satisfies

$$
\dot{x}=\alpha f(x)+(1-\alpha) g(x),
$$

with $\alpha=\alpha(x)$ given in (63). Substituting $\alpha(x)$ in (64) we get

$$
\dot{x}=\frac{A_{f g}(x) \eta}{\left(g_{2}(x)-\beta g_{1}(x)\right)-\left(f_{2}(x)-\beta f_{1}(x)\right)},
$$

where $A_{f g}(x)$ is given by

$$
A_{f g}(x)=\gamma_{1} \gamma_{\ell}\left\{x^{T} P\left(\phi^{f}-\phi^{g}\right)+\phi^{f^{T}} P \phi^{g}\right\}
$$

with $P=\left[\begin{array}{cc}0 & 1 \\ -1 & 0\end{array}\right]$ so that $x^{T} P x=0$ for all $x \in \mathbb{R}^{2}$. A point $\phi_{s} \in L_{s}$ is a singular equilibrium of $(23)$ if it satisfies $A_{f g}\left(\phi_{s}\right)=0$. The equation $A_{f g}(x)=0$ is satisfied by both focal points, i.e. $A_{f g}\left(\phi^{f}\right)=A_{f g}\left(\phi^{g}\right)=$ 0 , and so the curve

$$
L_{\phi}=\left\{x \in \mathbb{R}_{\geq 0}^{2}: A_{f g}(x)=0\right\},
$$

is the line containing both focal points. We thus conclude that any singular equilibrium must be located at $\phi_{s}=L_{\phi} \cap L_{s}$. The stability of $\phi_{s}$ follows by examining the direction of the vector field in (65). We know that

$$
\left(g_{2}(x)-\beta g_{1}(x)\right)-\left(f_{2}(x)-\beta f_{1}(x)\right)>0,
$$

for all $x \in L_{s}$, and hence the direction of the right-hand side of (65) depends only on the sign of $A_{f g}(x)$ along $L_{s}$. The function $A_{f g}(x)$ evaluated along $L_{s}$ (i.e. when $x=x_{1} \cdot \eta$ ) defines a line

$$
\left.A_{f g}(x)\right|_{x \in L_{s}}=\gamma_{1} \gamma_{\ell}\left\{\left\langle\phi^{f}-\phi^{g}, \eta^{\perp}\right\rangle x_{1}+\phi^{f^{T}} P \phi^{g}\right\}
$$

with slope

$$
\left.\frac{\partial}{\partial x_{1}} A_{f g}(x)\right|_{x \in L_{s}}=\gamma_{1} \gamma_{\ell}\left\langle\phi^{f}-\phi^{g}, \eta^{\perp}\right\rangle
$$

Note that the line in (69) is transversal to the line $L_{\phi}$ and they intersect at $\phi_{s}$ (because $A_{f g}\left(\phi_{s}\right)=0$ and $\left.\phi_{s} \in L_{s}\right)$. Therefore $A_{f g}(x)$ changes sign at $x=\phi_{s}$, so the local stability of $\phi_{s}$ depends on the sign of the slope in (70); namely

$$
\phi_{s} \text { is }\left\{\begin{array}{ll}
\text { stable } & \text { if }\left\langle\phi^{f}-\phi^{g}, \eta^{\perp}\right\rangle<0 \\
\text { unstable } & \text { if }\left\langle\phi^{f}-\phi^{g}, \eta^{\perp}\right\rangle>0
\end{array},\right.
$$

which are equivalent to the angle conditions in (31)-(32) (note that $\left\langle\phi^{f}-\phi^{g}, \eta^{\perp}\right\rangle \neq 0$ since $\phi^{f}, \phi^{g} \notin S$ by assumption). 


\section{B.3 Proof of Theorem 3.}

In what follows we restrict the proof to the case where $\gamma_{1}>\gamma_{\ell}$ (shown in Figure 6), but the converse case can be treated analogously. To construct the Poincaré map from $S^{f}$ onto itself, we first note that every point on $S^{f}$ satisfies $x_{2}=\beta x_{1}$, and so it is enough to analyze a one-dimensional Poincaré map that maps the segment $S_{1}^{f}$ onto itself. We write this map, $P$, as the composition of two scalar functions:

$$
\begin{aligned}
P: S_{1}^{f} & \rightarrow S_{1}^{f}, \\
r & \mapsto P_{B} \circ P_{A}(r),
\end{aligned}
$$

where

$$
\begin{aligned}
P_{A}: S_{1}^{f} & \rightarrow S_{1}^{g} \\
r & \mapsto P_{A}(r), \\
P_{B}: S_{1}^{g} & \rightarrow S_{1}^{f} \\
r & \mapsto P_{B}(r),
\end{aligned}
$$

The function $P_{A}$ maps a point in $S_{1}^{f}$ onto a "hit-point" in the segment $S_{1}^{g}$, whereas the function $P_{B}$ maps points in $S_{1}^{g}$ back onto a hit-point in $S_{1}^{f}$. In the cones $D_{f}$ and $D_{g}$ the trajectories follow standard linear dynamics, and therefore $P_{A}$ and $P_{B}$ can be written as

$$
\begin{aligned}
& P_{A}(r)=r e^{-\gamma_{1} T_{A}(r)}+\phi_{1}^{g}\left(1-e^{-\gamma_{1} T_{A}(r)}\right) \\
& P_{B}(r)=r e^{-\gamma_{1} T_{B}(r)}+\phi_{1}^{f}\left(1-e^{-\gamma_{1} T_{B}(r)}\right),
\end{aligned}
$$

whereby the functions $T_{A}(r)$ and $T_{B}(r)$ are the time it takes a trajectory starting in $r \in S^{f}$ (resp. $r \in S^{g}$ ) to hit the segment $S^{g}$ (resp. $\left.S^{f}\right)$. Next we proceed by parts showing that:

i. the set $S_{1}^{f}$ is invariant under the Poincaré map,

ii. the map is continuous in $S_{1}^{f}$,

iii. the map is non-decreasing in $S_{1}^{f}$, and

iv. the map is convex in $S_{1}^{f}$.

We will then show that these four statements imply that the Poincaré map has a unique stable fixed-point and hence, our statement holds.

\section{B.3.1 Invariance.}

From the qualitative analysis of Figure 6 we see that

$$
P_{A}\left(S_{1}^{f}\right) \in S_{1}^{g} \text {, and } P_{B}\left(S_{1}^{g}\right) \in S_{1}^{f},
$$

which together imply that $S_{1}^{f}$ is invariant under the map $P=P_{B} \circ P_{A}$. 


\section{B.3.2 Continuity.}

From the definitions in (74)-(75), the maps $P_{A}$ and $P_{B}$ are continuous in the time-to-hit, $T_{A}$ and $T_{B}$, and therefore it suffices to show that both $T_{A}(r)$ and $T_{B}(r)$ are continuous for $r \in S_{1}^{f}$ and $r \in S_{1}^{g}$, respectively. Starting from $x(0)=x^{0} \in S^{f}$, at time $t=T_{A}$ the state is

$$
\begin{aligned}
& x_{1}\left(T_{A}\right)=x_{1}^{0} e^{-\gamma_{1} T_{A}}+\phi_{1}^{g}\left(1-e^{-\gamma_{1} T_{A}}\right), \\
& x_{2}\left(T_{A}\right)=x_{2}^{0} e^{-\gamma_{\ell} T_{A}}+\phi_{2}^{g}\left(1-e^{-\gamma_{\ell} T_{A}}\right) .
\end{aligned}
$$

If we impose the condition that $x\left(T_{A}\right) \in S^{g}$ (i.e. $x_{2}\left(T_{A}\right)=\beta x_{1}\left(T_{A}\right)$ ), then we get an implicit equation for the time-to-hit $T_{A}(r)$ :

$$
F_{A}\left(T_{A}, r\right)=\left(\phi_{2}^{g}-\beta r\right)\left(1-e^{-\gamma_{\ell} T_{A}}\right)-\beta\left(\phi_{1}^{g}-r\right)\left(1-e^{-\gamma_{1} T_{A}}\right) \equiv 0,
$$

with $r \in S_{1}^{f}$. Likewise, we can obtain an implicit equation for the time-to-hit $T_{B}$ :

$$
F_{B}\left(T_{B}, r\right)=\left(\phi_{2}^{f}-\beta r\right)\left(1-e^{-\gamma_{\ell} T_{B}}\right)-\beta\left(\phi_{1}^{f}-r\right)\left(1-e^{-\gamma_{1} T_{B}}\right) \equiv 0,
$$

with $r \in S_{1}^{g}$. Both equations, $F_{A}\left(T_{A}, r\right) \equiv 0$ and $F_{A}\left(T_{A}, r\right) \equiv 0$, have a unique non-trivial solution $T_{A}=T_{A}(r)$ and $T_{B}=T_{B}(r)$ (see Figure 6), and therefore we need to show that these are continuous for $r \in S_{1}^{f}$ and $r \in S_{1}^{g}$, respectively. We will prove this only for $T_{A}(r)$ (the case of $T_{B}(r)$ can be shown with symmetrical arguments). Rewrite the implicit equation in (77) as

$$
\tilde{G}_{A}(r)=G\left(T_{A}(r)\right)
$$

with the functions

$$
\begin{aligned}
\tilde{G}_{A}(r) & =\frac{\phi_{2}^{g}-\beta r}{\beta\left(\phi_{1}^{g}-r\right)}, r \in S_{1}^{f}, \\
G\left(T_{A}\right) & =\frac{1-e^{-\gamma_{1} T_{A}}}{1-e^{-\gamma_{\ell} T_{A}}}, T_{A}>0 .
\end{aligned}
$$

It is easy to see that $\tilde{G}_{A}(r)$ is continuous in $S_{1}^{f}$ (because $\left(\phi_{1}^{g}-r\right)>0$ for $r \in S_{1}^{f}$, see Figure 6 ) and $G\left(T_{A}\right)$ is continuous for $T_{A}>0$. Moreover, the derivative of $G$ is

$$
\frac{\mathrm{d} G}{\mathrm{~d} T_{A}}=\frac{N\left(T_{A}\right)}{D\left(T_{A}\right)}=\frac{\gamma_{1} e^{-\gamma_{1} T_{A}}\left(1-e^{-\gamma_{\ell} T_{A}}\right)-\gamma_{\ell} e^{-\gamma_{\ell} T_{A}}\left(1-e^{-\gamma_{1} T_{A}}\right)}{\left(1-e^{-\gamma_{\ell} T_{A}}\right)^{2}},
$$

where

$$
N\left(T_{A}\right)=\gamma_{1} \gamma_{\ell} e^{-\gamma_{1} T_{A}} e^{-\gamma_{\ell} T_{A}}\left(N_{2}\left(T_{A}\right)-N_{1}\left(T_{A}\right)\right)
$$

with $N_{1}\left(T_{A}\right)=\left(e^{\gamma_{1} T_{A}}-1\right) / \gamma_{1}$ and $N_{2}\left(T_{A}\right)=\left(e^{\gamma_{\ell} T_{A}}-1\right) / \gamma_{\ell}$. Since $\gamma_{1}>\gamma_{\ell}$, we have that $N_{1}\left(T_{A}\right)>N_{2}\left(T_{A}\right)$ for all $T_{A}>0$, and consequently $\mathrm{d} G / \mathrm{d} T_{A}<0$ for all $T_{A}>0$. The function $G\left(T_{A}\right)$ is then continuous and strictly decreasing for $T_{A}>0$ and, therefore, admits a well-defined, continuous and strictly decreasing inverse function $H \circ G\left(T_{A}\right)=T_{A}$. From the implicit equation in (78) we then get the time-to-hit as

$$
T_{A}(r)=H \circ \tilde{G}_{A}(r),
$$

which is a composition of two continuous functions, and hence continuous. This implies that the Poincaré map is continuous for $r \in S_{1}^{f}$. 


\section{B.3.3 Monotonicity.}

By the chain rule the derivative of $P=P_{B} \circ P_{A}$ is

$$
P^{\prime}(r)=P_{B}^{\prime}\left(P_{A}(r)\right) \cdot P_{A}^{\prime}(r) \text {. }
$$

A sufficient condition for $P(r)$ to be non-decreasing is that both $P_{A}$ and $P_{B}$ are non-increasing, i.e. $\mathrm{d} P_{A} / \mathrm{d} r \leq$ 0 for $r \in S_{1}^{f}$ and $\mathrm{d} P_{B} / \mathrm{d} r \leq 0$ for $r \in S_{1}^{g}$. These two statements can be proven by contradiction. If $\mathrm{d} P_{A} / \mathrm{d} r>0$ for $r \in \mathcal{R} \subseteq S_{1}^{f}$, two trajectories starting at different points in the projection of $\mathcal{R}$ onto $S^{f}$ would intersect in the cone $D_{g}$, which is a contradiction because in $D_{g}$ the vector field is uniquely defined. With the same argument, one concludes that $\mathrm{d} P_{B} / \mathrm{d} r \leq 0$ for $r \in S_{1}^{g}$, and hence $P^{\prime}(r) \geq 0$ for $r \in S_{1}^{f}$.

\section{B.3.4 Convexity.}

By the chain rule we get

$$
P^{\prime \prime}(r)=P_{B}^{\prime \prime}\left(P_{A}(r)\right)\left(P_{A}^{\prime}(r)\right)^{2}+P_{B}^{\prime}\left(P_{A}(r)\right) P_{A}^{\prime \prime}(r),
$$

so that $P(r)$ is convex in $S_{1}^{f}$ if three conditions hold: (a) $P_{B}(r)$ is non-increasing in $S_{1}^{g}$, (b) $P_{A}(r)$ is concave in $S_{1}^{f}$, and (c) $P_{B}(r)$ is convex in $S_{1}^{g}$. From the previous part of the proof we already know that condition (a) holds. Next we show that conditions (b)-(c) are also satisfied.

From the definition of $P_{A}$ in (74), its first and second derivatives can be written as

$$
\begin{aligned}
& P_{A}^{\prime}(r)=e^{-\gamma_{1} T_{A}(r)}\left(1+\gamma_{1} T_{A}^{\prime}(r)\left(\phi_{1}^{g}-r\right)\right), \\
& P_{A}^{\prime \prime}(r)=-\gamma_{1} T_{A}^{\prime}(r) P_{A}^{\prime}(r)+\gamma_{1} e^{-\gamma_{1} T_{A}(r)}\left\{\left(\phi_{1}^{g}-r\right) T_{A}^{\prime \prime}(r)-T_{A}^{\prime}(r)\right\},
\end{aligned}
$$

whereas the derivative of $T_{A}$ can be obtained using the chain rule in the relation (78):

$$
T_{A}^{\prime}(r)=\left(\frac{1}{G^{\prime}\left(T_{A}(r)\right)}\right) \frac{\phi_{2}^{g}-\beta \phi_{1}^{g}}{\beta\left(\phi_{1}^{g}-r\right)^{2}},
$$

with $G\left(T_{A}\right)$ defined in (80). After some manipulations the second derivative of $T_{A}$ can be written as

$$
T_{A}^{\prime \prime}(r)=T_{A}^{\prime}(r)\left(\frac{2}{\phi_{1}^{g}-r}-T_{A}^{\prime}(r) F\left(T_{A}(r)\right)\right),
$$

where we have defined the function $F\left(T_{A}\right)=G^{\prime \prime}\left(T_{A}\right) / G^{\prime}\left(T_{A}\right)$. Substitution of $P_{A}^{\prime}$ and $T_{A}^{\prime \prime}$ in the expression for $P_{A}^{\prime \prime}$ (eq. (86)) yields

$$
P_{A}^{\prime \prime}(r)=-\gamma_{1}\left(T_{A}^{\prime}(r)\right)^{2} e^{-\gamma_{1} T_{A}(r)}\left(\phi_{1}^{g}-r\right) \cdot\left(\gamma_{1}+F\left(T_{A}(r)\right)\right) .
$$

Using $G\left(T_{A}\right)$ in (80) we can show that

$$
\gamma_{1}+F=\gamma_{1}+\frac{\gamma_{\ell} N_{1}-\gamma_{1} N_{2}}{N_{2}-N_{1}}-\frac{2}{N_{2}}
$$

with $N_{1}$ and $N_{2}$ defined previously in (81). Now define the function

$$
Q=\left(\gamma_{1}+F\right) N_{2}\left(N_{1}-N_{2}\right),
$$

which has the same sign as $\left(\gamma_{1}+F\right)$ because $N_{2}>0$ for $T_{A}>0$, and $N_{1}>N_{2}$ for $r>0$ and $\gamma_{1}>\gamma_{\ell}$. After substituting $N_{1}$ and $N_{2}, Q$ becomes

$$
Q\left(T_{A}\right)=\left(\gamma_{1}-\gamma_{\ell}\right)\left(e^{\left(\gamma_{1}+\gamma_{\ell}\right) T_{A}}-1\right)-\left(\gamma_{1}+\gamma_{\ell}\right)\left(e^{\gamma_{1} T_{A}}-e^{\gamma_{\ell} T_{A}}\right) .
$$


From (92) we see that $Q$ satisfies

$$
Q(0)=0, \text { and } \lim _{T_{A} \rightarrow \infty} Q\left(T_{A}\right)=\infty
$$

and is strictly increasing when $\gamma_{1}>\gamma_{\ell}$ :

$$
\frac{\mathrm{d} Q}{\mathrm{~d} T_{A}}=\left(\gamma_{1}+\gamma_{\ell}\right) \gamma_{1} \gamma_{\ell} e^{\left(\gamma_{1}+\gamma_{\ell}\right) T_{A}}\left(\frac{1-e^{-\gamma_{\ell} T_{A}}}{\gamma_{\ell}}-\frac{1-e^{-\gamma_{1} T_{A}}}{\gamma_{1}}\right)>0, T_{A}>0 .
$$

We thus conclude that $Q\left(T_{A}\right)>0$ for $T_{A}>0$, and so $\left(\gamma_{1}+F\left(T_{A}(r)\right)\right)>0$ for all $T_{A}>0$. In addition we know that $\left(\phi_{1}^{g}-r\right)>0$ for $r \in S_{1}^{f}$ (Figure 6), which from the expression for $P_{A}^{\prime \prime}$ in (89) implies that $P_{A}(r)$ is concave for $r \in S_{1}^{f}$ (i.e. $P_{A}^{\prime \prime}(r)<0$ for $r \in S_{f}$ ).

The proof that $P_{B}(r)$ is convex for $r \in S_{1}^{g}$ is to a large extent symmetrical to the one for concavity of $P_{A}$. For brevity we omit the intermediate steps and only point out the differences. The function $P_{B}(r)$ is defined in (75) and the time-to-hit $T_{B}$ is the solution of the implicit equation

$$
\tilde{G}_{B}(r)=G\left(T_{B}(r)\right)
$$

with $G$ defined in (80) and

$$
\tilde{G}_{B}(r)=\frac{\phi_{2}^{f}-\beta r}{\beta\left(\phi_{1}^{f}-r\right)} .
$$

The derivative of $T_{B}$ can be obtained using the chain rule (cf. (87)) in the relation (95):

$$
T_{B}^{\prime}(r)=\left(\frac{1}{G^{\prime}\left(T_{B}(r)\right)}\right) \frac{\phi_{2}^{f}-\beta \phi_{1}^{f}}{\beta\left(\phi_{1}^{f}-r\right)^{2}},
$$

so that the second derivative of $P_{B}$ can be written as

$$
P_{B}^{\prime \prime}(r)=-\gamma_{1}\left(T_{B}^{\prime}(r)\right)^{2} e^{-\gamma_{1} T_{B}(r)}\left(\phi_{1}^{f}-r\right) \cdot\left(\gamma_{1}+F\left(T_{B}(r)\right)\right),
$$

which can be obtained by following, mutatis mutandis, the steps in equations (85)-(89). Note that the form of $P_{B}^{\prime \prime}$ is symmetrical to $P_{A}^{\prime \prime}$ in (87). We have already established that $\left(\gamma_{1}+F\left(T_{B}(r)\right)\right)>0$ for $T_{B}>0$, but in this case the term $\left(\phi_{1}^{f}-r\right)<0$ because $r \in S_{1}^{g}$ (see Figure 6). We conclude that $P_{B}^{\prime \prime}(r)>0$ for $r \in S_{1}^{g}$ and thus $P_{B}$ is convex for $r \in S_{1}^{g}$.

\section{B.3.5 Fixed point.}

A stable fixed point of the Poincaré map, i.e. a point $r^{*}$ such that $P\left(r^{*}\right)=r^{*}$ with $(\mathrm{d} P / \mathrm{d} r)\left(r^{*}\right)<1$, indicates a stable periodic orbit passing through $r^{*}$. We will show that $P$ has a unique stable fixed point in $S_{1}^{f}=\left[\phi_{1}^{f}, x_{1}^{f}\right]$. We first analyze the Poincaré map at the endpoints of the segment $S_{1}^{f}$. The image of the segment $S_{1}^{g}$ under the map $P_{B}$ satisfies

$$
P_{B}\left(S_{1}^{g}\right)>\phi_{1}^{f}
$$

because in $D_{f}$ the coordinate $x_{1}$ can only reach $\phi_{1}^{f}$ asymptotically (when time tends to infinity, see Figure $6)$. Now, since $P_{A}\left(\phi_{1}^{f}\right) \in S_{1}^{g}$, (99) implies that

$$
P\left(\phi_{1}^{f}\right)>\phi_{1}^{f} .
$$


We also know that $S_{1}^{f}$ is invariant under $P$, and hence for the other endpoint, $r=x_{1}^{f}$, we have that

$$
P\left(x_{1}^{f}\right) \leq x_{1}^{f} .
$$

Since $P$ is a continuous map defined on a bounded and invariant set $\left(S_{1}^{f}\right)$, it must have at least one fixed point in $S_{1}^{f}$. Moreover, $P$ is also convex and therefore it can have at most two fixed points (these can be seen as intersections between the curve $y=P(r)$ and the identity line $y=r$, and therefore more than two fixed points would require $P$ to be concave in some interval, see Figure 12). From (100)-(101) we identify two cases:

(a) One fixed point. If (101) holds as strict inequality, we have a unique fixed point at $\phi_{1}^{f}<r^{*}<x_{1}^{f}$. Moreover, by (100) we know that $P(r)$ starts above the identity line, so that (see Figure 12)

$$
\left.\frac{\mathrm{d} P}{\mathrm{~d} r}\right|_{r=r^{*}}<1,
$$

and therefore the fixed point at $r^{*}$ is stable.

(b) Two fixed points. If (101) holds as equality, then the endpoint $x_{1}^{f}$ is also a fixed point. Now, if $r^{*}=x_{1}^{f}$ then both fixed points match and we have a unique stable fixed point, whereas if $r^{*} \neq x_{1}^{f}$, by the mean value theorem, monotonicity, and convexity of $P(r)$ it follows that

$$
\left.\frac{\mathrm{d} P}{\mathrm{~d} r}\right|_{r=x_{1}^{f}}>1,
$$

so that the fixed point at the endpoint is unstable.

We therefore conclude that the Poincaré map has a unique stable fixed point, which completes the proof.

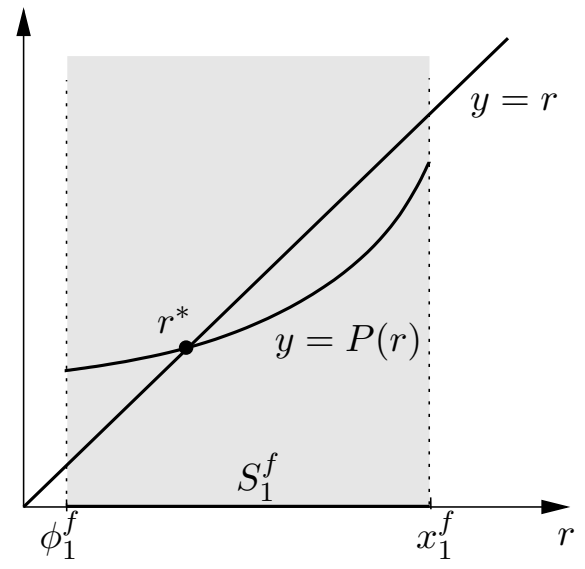

Figure 12: Schematic plot of a continuous, non-decreasing and convex Poincaré map and its stable fixed point. 


\section{B.4 Proof of Corollary 1.}

This proof follows from the one for Theorem 3. We know the sets satisfy $L_{s}=L_{\bar{s}}=\phi_{s}$ when

$$
x_{1}^{f}=x_{1}^{g} .
$$

The idea behind the proof is to show that $r=x_{1}^{f}$ is the unique stable fixed point of the Poincaré map and therefore corresponds to a degenerate stable limit cycle.

Evaluating the function $\tilde{G}_{A}(r)$ in $(79)$ at the endpoint of $S_{1}^{f}$ we get (after substituting (27))

$$
\tilde{G}_{A}\left(x_{1}^{f}\right)=\tilde{G}_{A}\left(x_{1}^{g}\right)=\frac{\gamma_{1}}{\gamma_{\ell}} .
$$

In addition, the function $G\left(T_{A}\right)$ in (80) is continuous at $T_{A}=0$ and given by (using L'Hôpital's rule)

$$
G(0)=\frac{\gamma_{1}}{\gamma_{\ell}}
$$

Equation (106) implies that the inverse of $G$ (defined by $H \circ G\left(T_{A}\right)=T_{A}$ ) satisfies $H\left(\gamma_{1} / \gamma_{\ell}\right)=0$. We can then combine (105)-(106) to establish that the first time-to-hit in (82) satisfies

$$
T_{A}\left(x_{1}^{f}\right)=0,
$$

which implies that $P_{A}\left(x_{1}^{f}\right)=x_{1}^{f}$. Conversely, we can also show that $P_{B}\left(x_{1}^{f}\right)=x_{1}^{f}$, and therefore $x_{1}^{f}$ is itself a fixed point of the Poincaré map $P=P_{B} \circ P_{A}$. Note that from (85) we obtain

$$
\left.P^{\prime}(r)\right|_{r=x_{1}^{f}}=1
$$

which implies that $r^{*}=x_{1}^{f}$ is the only fixed point (by the same arguments as in case (b) of Section B.3.5, existence of two fixed points would imply that $P^{\prime}\left(x_{1}^{f}\right)>1$, which contradicts (108)). Stability follows from the monotonicity and convexity of $P$. Therefore, $r^{*}=x_{1}^{f}$ is the unique stable fixed point of $P$ (see the analysis before Figure 12), and thus it corresponds to a degenerate stable oscillation collapsed to the point $\left(x_{1}^{f}, \beta x_{1}^{f}\right)$. 